\title{
A multiscale Darcy-Brinkman model for fluid flow in fractured porous media
}

\author{
Matteo Lesinigo • Carlo D’Angelo • \\ Alfio Quarteroni
}

Received: 29 September 2009 / Revised: 29 September 2010 / Published online: 28 October 2010 (C) Springer-Verlag 2010

\begin{abstract}
The aim of this work is to present a reduced mathematical model for describing fluid flow in porous media featuring open channels or fractures. The Darcy's law is assumed in the porous domain while the Stokes-Brinkman equations are considered in the fractures. We address the case of fractures whose thickness is very small compared to the characteristic diameter of the computational domain, and describe the fracture as if it were an interface between porous regions. We derive the corresponding interface model governing the fluid flow in the fracture and in the porous media, and establish the well-posedness of the coupled problem. Further, we introduce a finite element scheme for the approximation of the coupled problem, and discuss solution strategies. We conclude by showing the numerical results related to several test cases and compare the accuracy of the reduced model compared with the non-reduced one.
\end{abstract}

Mathematics Subject Classification (2000) 76D07 · 76S05 · 35Q86 · 65L60

\section{Introduction}

Reduced models of fluid flow in fractured media have an intrinsic interest due to their potential applications in different domains, and have been studied by a number

M. Lesinigo $(\varangle) \cdot$ A. Quarteroni

SB-MATHICSE-CMCS, École Polytechnique Fédérale de Lausanne (EPFL),

Station 8, 1015 Lausanne, Switzerland

e-mail: matteo.lesinigo@epfl.ch

A. Quarteroni

e-mail: alfio.quarteroni@epfl.ch

C. D'Angelo · A. Quarteroni

MOX-Modellistica e Calcolo Scientifico, Dipartimento di Matematica,

Politecnico di Milano, via Bonardi 9, 20133 Milan, Italy

e-mail: carlo.dangelo@polimi.it 
of authors; see, for example, [2,21,24,30] and references therein. Typically, Darcy's equations are used to model the fluid flow in the porous media, a heterogeneous $N$-dimensional domain, made of regions featuring different permeabilities. Thin regions in the form of "inclusions" are called fractures; in the numerical approximation they might require excessive mesh refining and increased computational costs when treated as fully $N$ dimensional regions. For this reason, reduced models in which fractures are represented as $N-1$ manifolds are often used; then suitable fracture flow equations are derived and coupled with classical porous media flow equations [21,25]. Such model reduction is obviously intended to represent the heterogeneity of the permeability field; in fact, the basic model of both the porous domain and the fracture domain is the Darcy's model.

In this work, we focus on highly-permeable or even open fractures, in which flow can be described by the Stokes-Brinkman equations: more specifically, we address the coupling of a Darcy model for fluid flow in a porous domain, with a reduced Stokes-Brinkman model of fluid flow within a fracture of the domain. This problem has obvious applications in fields like geophysics or hydrogeology. For instance, it may describe hydrocarbon migration, or groundwater flows, or more generally twophase flows in fractured soils [7]. The intrinsic interest in having both accurate and computationally cheap models describing such phenomena is still promoting the investigation of advanced numerical techniques. Fractures can substantially modify the flow pattern, acting as either barriers or highly permeable channels, in which most of the fluid is collected and redistributed. Recent works [3] confirm that reduced models can accurately capture such effects. Finally, let us mention that incompressible flow models are used also in medicine to describe tissue perfusion, transport of nutrients from arterial vessels to cells, or heat transfer with application to hyperthermia [15] and other clinical treatments [20].

Often, transverse dimensions of fractures or vessels are negligible when compared to the dimensions of the considered porous matrices. For this reason geometrical multiscale models treating fractures or vessels as entities with lower dimension are very interesting. Those reduced models may lead to significant computational advantages as it is not necessary to solve the full Brinkman model at the small spatial scale in the fracture or in the vessel. Moreover, our approach makes it possible to treat different geometrical multiscale couplings, such as the classical 2D porous domain with a 1D fracture (see Fig. 1a), a 3D porous domain with a 2D fracture (see Fig. 1b) or a more exotic 3D porous domain with a 1D fracture (see Fig. 1c), modeling, for instance, blood vessels surrounded by biological tissues [13,20].

We warn the reader that the distinction between "fracture" and "vessel" uniquely refers to the geometry of the problem (see Fig. 1). More particularly, in both cases we consider a direct interaction between a free fluid and a porous medium. Therefore the effects that, e.g., the physiological structure of blood vessel (multiple layers of poro-elastic materials) have on the fluid motion are not taken into account. The only difference between the two situations is that in the case of the fracture the reduced model lives on a bi-dimensional surface (2D/3D coupling) whereas in the case of the vessel it is one-dimensional and lives on the mean line of the vessel (1D/3D coupling).

The paper is organized as follows. In Sect. 2 we provide the basic equations of our mathematical model. In Sect. 3 we derive the reduced model for a fracture in a $2 \mathrm{D}$ 


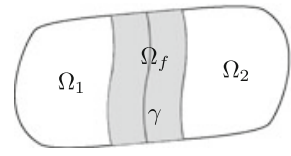

(a)

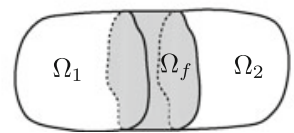

(b)

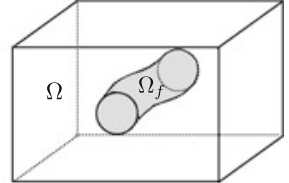

(c)

Fig. 1 Reference geometries for the problems considered. a 1D fracture in a 2D domain. b 2D fracture in a $3 \mathrm{D}$ domain. $\mathrm{c} 1 \mathrm{D}$ vessel in a $3 \mathrm{D}$ domain

domain, and present the analysis of the coupled problem. In Sect. 4 we briefly explain how the model can be extended to the case of a planar fracture in a 3D domain or of a vessel in a 3D domain. Section 5 is devoted to the numerical approximation of the reduced coupled problem while in Sect. 7 we provide numerical simulations on a test case. Finally we present some conclusions in Sect. 8.

\section{The coupled Darcy-Brinkman model}

In this introductory section we wish to briefly recall the Darcy and the Brinkman model, and discuss the coupling conditions.

The Darcy model is often used to describe the motion of an incompressible fluid in a porous medium. Indeed, it is a widely accepted approximation of the filtration problem for low velocity flow in which inertial effects can be neglected. It can be written as:

$$
\begin{aligned}
\mathbf{u} & =-\mathrm{K} \nabla p & & \text { in } \Omega_{p}, \\
\nabla \cdot \mathbf{u} & =q & & \text { in } \Omega_{p},
\end{aligned}
$$

where $\Omega_{p}$ is the domain occupied by the porous media, $\mathrm{K}$ is a bounded symmetric positive definite permeability tensor, and $\mathbf{u}$ and $p$ are respectively the velocity and the pressure in the porous medium. Equation (2.1a) is the Darcy law (see [14]) while (2.1b) is the equation of mass balance. Usually the source term $q$ is equal to 0 .

The Brinkman model we will use to describe the flow motion in the fracture or in the vessel is instead a generalization of the Stokes model that represents a valid approximation of the Navier-Stokes equations at low Reynolds numbers. It reads:

$$
\begin{aligned}
\beta \mathbf{u}-\varepsilon \Delta \mathbf{u}+\nabla p=\mathbf{f} & \text { in } \Omega_{f}, \\
\nabla \cdot \mathbf{u}=0 & \text { in } \Omega_{f},
\end{aligned}
$$

where $\Omega_{f}$ is the domain that describes the fracture, $\mathbf{u}$ and $p$ represent the velocity and the pressure in the fracture, respectively, $\beta$ is a scalar, $\varepsilon$ is the inverse of the Reynolds number and $\mathbf{f}$ is a momentum source term. More precisely, we are interested in a slightly different formulation in which the scalar $\beta$ is replaced by $\mathrm{K}_{f}^{-1}$, the inverse of a permeability tensor and in which a mass source term can be present in Eq. (2.2b), 
i.e.:

$$
\begin{aligned}
\mathbf{K}_{f}^{-1} \mathbf{u}-\varepsilon \Delta \mathbf{u}+\nabla p=\mathbf{f} & & \text { in } \Omega_{f}, \\
\nabla \cdot \mathbf{u}=g & & \text { in } \Omega_{f} .
\end{aligned}
$$

The model (2.3) is interesting because it contains as special cases both the Darcy and the Brinkman model.

Remark 1 Equations (2.3) arise in the time discretization of the classical Stokes model with $\mathrm{K}_{f}=\mathrm{I} \Delta t$, being $\mathrm{I}$ the identity matrix and $\Delta t$ the time step.

When coupling the (2.1) with (2.3), suitable coupling conditions have to be provided at the interface, say $\Gamma_{D B}$, of the two subregions in order to ensure the well-posedness of the coupled problem. A first classical choice is to require the normal flux to be continuous:

$$
\mathbf{u}_{D} \cdot \mathbf{n}_{D}=\mathbf{u}_{B} \cdot \mathbf{n}_{D} \quad \text { on } \Gamma_{D B}
$$

where $\mathbf{u}_{D}$ is the Darcy velocity at the interface, $\mathbf{u}_{B}$ the Brinkman one and $\mathbf{n}_{D}$ is the external normal unit vector to the Darcy domain. Equation (2.4) is not sufficient to close the model. Many proposals for additional coupling conditions have been made; in this paper we consider a variant of the well known Beavers, Joseph and Saffman conditions [5,39]. Starting from the works by Jager and Mikelic [26] and Discacciati and Quarteroni [16], we first simplify the Beavers, Joseph and Saffman conditions. Let us suppose that the interface $\Gamma_{D B}$ is smooth enough to allow for the definition of a unique unit vector $\mathbf{n}_{S}$ perpendicular to the interface and oriented from the fracture towards the porous medium. Introducing also a generic unit vector $\boldsymbol{\tau}$ belonging to the uniquely defined tangential plane at a generic point of fracture-medium interface, the simplified Beavers, Joseph and Saffman equations may be written as:

$$
\begin{aligned}
\varepsilon \frac{\partial \mathbf{u}_{B}}{\partial \mathbf{n}_{B}} \cdot \boldsymbol{\tau} & =0 & & \text { on } \Gamma_{D B}, \\
p_{B}-\varepsilon \frac{\partial \mathbf{u}_{B}}{\partial \mathbf{n}_{B}} \cdot \mathbf{n}_{B} & =p_{D} & & \text { on } \Gamma_{D B},
\end{aligned}
$$

where the subscript $D$ stands for quantity to refer to the Darcy model, $B$ to the Brinkman one. In particular, tangential viscous losses are neglected in (2.5a), whose general form is $\varepsilon \frac{\partial \mathbf{u}_{B}}{\partial \mathbf{n}_{B}} \cdot \boldsymbol{\tau}=\alpha \mathbf{u}_{B} \cdot \boldsymbol{\tau}, \alpha>0$. However, this simplification does not alter the generality of the presentation. Moving from this set of equations, in the first part of this work, we will derive a reduced model in which the fracture problem is averaged along the cross-sections of the fracture and is replaced by a lower dimensional problem. Such technique applies to the case of a bi-dimensional (or tri-dimensional) porous domain with a thin fracture, which yields after model reduction a 2D-1D (or 3D-2D) coupled problem, or to the case of a three-dimensional porous domain including a vessel fracture, giving rise to a reduced 3D-1D coupled model. In each case, the strategy that we adopt to obtain a reduced model for the coupled problem (2.1), (2.3), (2.4) and (2.5) can be schematically summarized through the following steps: 
Fig. 2 Domains, boundaries and interfaces
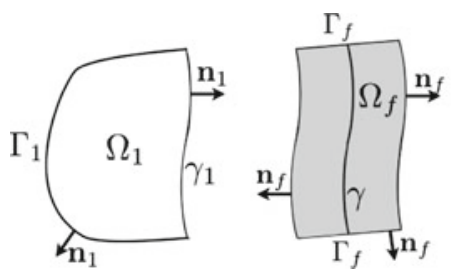

(i) At every point of the average line/surface of the fracture or vessel, introduce a local orthonormal reference system.

(ii) Project the Brinkman equations on the coordinate system introduced at step (i).

(iii) Average the resulting equations over every cross section.

(iv) Introduce suitable closure conditions based on convenient assumptions on the profile of pressure and normal flux in the fracture or in the vessel.

(v) Obtain the weak formulation of the problem by eliminating the flux on the Darcy-Brinkman interface with the closure conditions introduced at step (iv).

After the derivation of the reduced model, we will investigate its well-posedness, then we will present some numerical results, showing that the relevant features of fluid flow in a fractured porous domain are well captured by our model.

\section{A fracture in a bi-dimensional domain}

In this section we provide a detailed description of the reduced model for a fracture in a bi-dimensional domain.

\subsection{Geometry of the problem}

Let $\Omega \subset \mathbb{R}^{2}$ be a bounded domain consisting of three open bi-dimensional subsets $\Omega_{1}, \Omega_{2}$ and $\Omega_{f}$ such that $\bar{\Omega}=\overline{\Omega_{1}} \cup \overline{\Omega_{2}} \cup \overline{\Omega_{f}}$ (see Fig. 1a). Moreover suppose that $\Omega_{f}$ separates $\Omega_{1}$ from $\Omega_{2}$. Under these assumptions $\Omega_{1} \cap \Omega_{f}=\emptyset, \Omega_{2} \cap \Omega_{f}=\emptyset$ and $\overline{\Omega_{1}} \cap \overline{\Omega_{2}}=\emptyset$. In particular, $\Omega_{f}$ corresponds to the fracture while $\Omega_{1}$ and $\Omega_{2}$ correspond to two regions occupied by the porous medium. We also suppose that $\partial \Omega_{f}$ and $\partial \Omega_{i}$ are Lipschitz continuous boundaries. We call the Stokes-Brinkman interfaces $\gamma_{1}=\overline{\Omega_{1}} \cap \overline{\Omega_{f}}$ and $\gamma_{2}=\overline{\Omega_{2}} \cap \overline{\Omega_{f}}$. We denote by $\Gamma_{1}$ the remaining part of $\partial \Omega_{1}$, with $\Gamma_{2}$ the remaining part of $\partial \Omega_{2}$ and with $\Gamma_{f}$ the remaining part of $\partial \Omega_{f}$. We denote with $\Gamma_{1, D}$ the part of $\Gamma_{1}$ where a Dirichlet boundary condition is assigned and $\Gamma_{1, N}$ that where a Neumann boundary condition is given. Same considerations hold for $\Gamma_{2}$ and $\Gamma_{f}$. Note that $\Gamma_{f}$ is made of two separate segments. We suppose that on each of these segments either a Dirichlet or a Neumann boundary condition is assigned (mixed Dirichlet/Neumann conditions on the same segment are not allowed). For the sake of simplicity we suppose that $\Gamma_{1, D}, \Gamma_{2, D}$ and $\Gamma_{f, D}$ are nonempty subsets of $\Gamma_{1}, \Gamma_{2}$ and $\Gamma_{f}$, respectively. Moreover we introduce $\mathbf{n}_{1}, \mathbf{n}_{2}$ and $\mathbf{n}_{f}$, the external normal unit vectors to $\Omega_{1}, \Omega_{2}$ and $\Omega_{f}$ respectively. Figure 2 shows the nomenclature introduced. 
Furthermore we suppose that the distance separating $\Omega_{1}$ from $\Omega_{2}$ is small with respect to the size of the porous media. In this sense we can think that $\Omega_{f}$ develops along a line $\gamma$. More precisely we can represent $\Omega_{f}=\left\{\mathbf{x} \in \mathbb{R}^{2}: \mathbf{x}=\mathbf{y}+\xi \frac{\delta}{2} \mathbf{n}\right\}$ where $\mathbf{y} \in \gamma, \delta$ is the width of $\Omega_{f}, \xi \in[-1 ; 1]$ and $\mathbf{n}$ is the unit vector normal to $\gamma$ at the point $\mathbf{y}$ directed from $\Omega_{1}$ to $\Omega_{2}{ }^{1}$. If $\gamma$ is smooth enough [41] we can introduce an intrinsic curvilinear reference system, whose variables are the arc length $s \in[0, L]$ (which uniquely identifies the points of $\gamma, L$ being the total length) and $\xi$. The corresponding unit vectors are the normal unit vector $\mathbf{n}$ and the tangential unit vector $\boldsymbol{\tau}$. At every point $s$ on $\gamma$, the unit vector $\mathbf{n}$ uniquely identifies a section of the subdomain $\Omega_{f}$. Moreover, at every $s, \mathbf{n}$ and $\boldsymbol{\tau}$ may be used to define a local orthogonal reference system. With little abuse of notation, in this work we denote by the same symbol different functions defined on $\gamma, \gamma_{1}$ or $\gamma_{2}$, if they have the same values at each section. For example, for a given function $f$ defined on one of the three lines, say $\gamma_{1}$, we may define a new function $\tilde{f}$ whose value at a given point of another line, say $\gamma_{2}$, is equal to the value of $f$ at the corresponding point at the same $s$-section. When it is clear from the context on which line such a function is defined, we may use the same symbol for $f$ and its "projection" $\tilde{f}$. Moreover, it is useful to refer the integral of $f$ on $\gamma_{1}$ to the integral of $\tilde{f}$ on $\gamma_{2}$, using the associated change of variables. Assume that there exists $\bar{r}>\delta / 2$ such that the curvature radius $r$ of $\gamma$ is larger than or equal to $\bar{r}$ for every $s$; then, the Jacobian $\mathrm{J}$ for such change of variable satisfies

$$
0<t_{\min }=\frac{r_{\min }-\delta / 2}{r_{\min }+\delta / 2} \leq|\mathrm{J}| \leq \frac{r_{\min }+\delta / 2}{r_{\min }-\delta / 2}=t_{\max },
$$

where $r_{\min }$ is the minimum curvature radius of $\gamma$.

Before getting into the details of the derivation of the reduced model, let us introduce some useful transformations. Let $\mathrm{R}$ be the orthonormal matrix mapping the canonical basis $\left[\mathbf{e}_{1}, \mathbf{e}_{2}\right]$ onto the local basis $[\mathbf{n}, \boldsymbol{\tau}]$, and its normal and tangential projectors:

$$
\mathrm{R}=\left[\begin{array}{l}
\mathbf{n}^{T} \\
\boldsymbol{\tau}^{T}
\end{array}\right]=\left[\begin{array}{l}
\mathbf{n}^{T} \\
\mathbf{0}^{T}
\end{array}\right]+\left[\begin{array}{l}
\mathbf{0}^{T} \\
\boldsymbol{\tau}^{T}
\end{array}\right]=\mathrm{N}+\mathrm{T}
$$

We will need to refer the differential operators to the local coordinates. Notice that, for a given scalar field $u$ and unit vector $\mathbf{v}$, we have $\partial u / \partial \mathbf{v}=\nabla u \cdot \mathbf{v}$ : hence, using this identity with $\mathbf{v}=\mathbf{n}$ and $\mathbf{v}=\boldsymbol{\tau}$, we get

$$
\frac{\partial u}{\partial \mathbf{n}} \mathbf{n}+\frac{\partial u}{\partial \boldsymbol{\tau}} \boldsymbol{\tau}=\left(\mathrm{R}^{T} \mathrm{R}\right) \nabla u=\nabla u
$$

Similarly, for a vector field $\mathbf{u}$, the trace of a tensor being invariant under changes of variables, we have

$$
\frac{\partial \mathbf{u}}{\partial \mathbf{n}} \cdot \mathbf{n}+\frac{\partial \mathbf{u}}{\partial \boldsymbol{\tau}} \cdot \boldsymbol{\tau}=\operatorname{trace}\left(\mathrm{RJR}^{T}\right)=\operatorname{trace}(J)=\nabla \cdot \mathbf{u}
$$

\footnotetext{
${ }^{1}$ The choice of the orientation is arbitrary.
} 
where $\mathbf{J}=\partial u_{i} / \partial x_{j}$ is the Jacobian matrix of $\mathbf{u}$. As regards second order operators of a scalar field, we observe that for each unit vector $\mathbf{v}$ we have $\partial^{2} u / \partial^{2} \mathbf{v}=\mathbf{v} \cdot \mathrm{Hv}$, where $\mathrm{H}=\partial^{2} u / \partial x_{i} \partial x_{j}$ is the Hessian matrix of $u$. Using this identity we have

$$
\frac{\partial^{2} u}{\partial^{2} \mathbf{n}}+\frac{\partial^{2} u}{\partial^{2} \boldsymbol{\tau}}=\operatorname{trace}\left(\mathrm{RHR}^{T}\right)=\operatorname{trace}(\mathrm{H})=\Delta u
$$

We point out that these relationships are only meant to refer the above differential operators to derivatives along orthogonal directions (n and $\boldsymbol{\tau}$, in our case) different from those of the canonical basis. In particular, we are not considering any change of variables. Instead, we will use such expressions in order to separate the normal and tangential components of the model variables in the derivation of the reduced model.

\subsection{Mathematical formulation of the original problem}

With the notations introduced in Sect. 3.1 the mathematical formulation of the full coupled problem reads as: find $\mathbf{u}, \mathbf{u}_{1}, \mathbf{u}_{2}, p, p_{1}$ and $p_{2}$ such that $(i=1,2)$ :

$$
\begin{aligned}
\mathbf{u}_{i} & =-\mathrm{K}_{i} \nabla p_{i} & & \text { in } \Omega_{i} \\
\nabla \cdot \mathbf{u}_{i} & =q_{i} & & \text { in } \Omega_{i} \\
\mathrm{~K}_{f}^{-1} \mathbf{u}-\varepsilon \Delta \mathbf{u}+\nabla p & =\mathbf{g} & & \text { in } \Omega_{f} \\
\nabla \cdot \mathbf{u} & =q & & \text { in } \Omega_{f} \\
\mathbf{u} \cdot \mathbf{n}_{i} & =\mathbf{u}_{i} \cdot \mathbf{n}_{i} & & \text { on } \gamma_{i} \\
-\varepsilon \frac{\partial \mathbf{u}}{\partial \mathbf{n}_{f}} \cdot \boldsymbol{\tau} & =0 & & \text { on } \gamma_{i}, \\
p-\varepsilon \frac{\partial \mathbf{u}}{\partial \mathbf{n}_{f}} \cdot \mathbf{n}_{f} & =p_{i} & & \text { on } \gamma_{i} \\
p_{i} & =\overline{p_{i}} & & \text { on } \Gamma_{i, D} \\
\mathrm{~K}_{i} \nabla p_{i} \cdot \mathbf{n}_{i} & =h_{i} & & \text { on } \Gamma_{i, N} \\
\mathbf{u} & =\overline{\mathbf{u}} & & \text { on } \Gamma_{f, D} \\
\varepsilon \frac{\partial \mathbf{u}}{\partial \mathbf{n}_{f}}-p \mathbf{n}_{f} & =\overline{\boldsymbol{h}} & & \text { on } \Gamma_{f, N}
\end{aligned}
$$

where $\bar{p}_{i}$ and $\overline{\mathbf{u}}$ are assigned Dirichlet boundary conditions and $h_{i}$ and $\overline{\boldsymbol{h}}$ are assigned Neumann boundary conditions. We denote by $\mathrm{K}_{i_{\max }}$ the maximum absolute value of the elements of the tensor $\mathrm{K}_{i}$ and $\lambda_{\min }\left(\mathrm{K}_{i}\right)$ the minimum eigenvalue of the same tensor which is strictly positive by assumption.

\subsection{Derivation of the reduced model}

As anticipated in Sect. 2, to obtain a reduced model we project the Brinkman equation in the fracture on the local orthogonal reference system at every $s$ on $\gamma$, and then we 
average the resulting equations on the corresponding $s$-cross-section of $\Omega_{f}$. Let us consider the mass conservation equation in $\Omega_{f}$,

$$
\nabla \cdot \mathbf{u}=q
$$

Projecting this equation on the reference system made of unit vectors $\mathbf{n}$ and $\boldsymbol{\tau}$ leads to:

$$
\frac{\partial \mathbf{u}}{\partial \mathbf{n}} \cdot \mathbf{n}+\frac{\partial \mathbf{u}}{\partial \boldsymbol{\tau}} \cdot \boldsymbol{\tau}=q
$$

Integrating the resulting equation over the segment $[\mathbf{y}-(\delta / 2) \mathbf{n}, \mathbf{y}+(\delta / 2) \mathbf{n}]$ where $\mathbf{y} \in \gamma$ yields:

$$
\left.\mathbf{u} \cdot \mathbf{n}\right|_{\gamma_{2}}-\left.\mathbf{u} \cdot \mathbf{n}\right|_{\gamma_{1}}+\delta \frac{\partial}{\partial \boldsymbol{\tau}} U_{\boldsymbol{\tau}}=\delta Q
$$

where we have set $U_{\boldsymbol{\tau}}=\frac{1}{\delta} \int_{-\delta / 2}^{\delta / 2} \mathbf{u} \cdot \boldsymbol{\tau} d \mathbf{n}$ and $Q=\frac{1}{\delta} \int_{-\delta / 2}^{\delta / 2} q d \mathbf{n}$. Finally, using Eq. (2.4) and given that $\frac{\partial}{\partial \tau}=\frac{\partial}{\partial s}$ we obtain a one-dimensional mass balance law on $\gamma$ with an additional term that accounts for the interaction with the Darcy problem in $\Omega_{1}$ and $\Omega_{2}$ :

$$
\left.\mathbf{u}_{1} \cdot \mathbf{n}_{1}\right|_{\gamma_{1}}+\left.\mathbf{u}_{2} \cdot \mathbf{n}_{2}\right|_{\gamma_{2}}=\delta\left(\frac{\partial}{\partial s} U_{\tau}-Q\right) \text { on } \gamma
$$

Remark 2 If the domain is fractureless (i.e. $\delta=0$ ) Eq. (3.3) states the continuity of the normal flux at the interface between the two porous media.

Let us apply the matrix R defined in (3.1) to Eq. (2.3a), yielding:

$$
\mathrm{RK}_{f}^{-1} \mathrm{R}^{T} \mathrm{Ru}-\varepsilon \mathrm{R} \Delta \mathbf{u}+\mathrm{R} \nabla p=\mathrm{Rg} .
$$

Given two vectors a and $\mathbf{b}$ in $\mathbb{R}^{2}$ we define $\mathbf{M}_{\mathbf{a b}}=\mathbf{a}^{T} \mathrm{~K}_{f}^{-1} \mathbf{b}$. Using such notation we can split Eq. (3.4) as:

$$
\begin{aligned}
& \mathbf{M}_{\mathbf{n n}}(\mathbf{u} \cdot \mathbf{n})+\mathbf{M}_{\mathbf{n} \boldsymbol{\tau}}(\mathbf{u} \cdot \boldsymbol{\tau})-\varepsilon \frac{\partial^{2} \mathbf{u}}{\partial \mathbf{n}^{2}} \cdot \mathbf{n}-\varepsilon \frac{\partial^{2} \mathbf{u}}{\partial \boldsymbol{\tau}^{2}} \cdot \mathbf{n}+\frac{\partial p}{\partial \mathbf{n}}=g_{\mathbf{n}}, \\
& \mathbf{M}_{\boldsymbol{\tau} \mathbf{n}}(\mathbf{u} \cdot \mathbf{n})+\mathbf{M}_{\boldsymbol{\tau} \boldsymbol{\tau}}(\mathbf{u} \cdot \boldsymbol{\tau})-\varepsilon \frac{\partial^{2} \mathbf{u}}{\partial \mathbf{n}^{2}} \cdot \boldsymbol{\tau}-\varepsilon \frac{\partial^{2} \mathbf{u}}{\partial \boldsymbol{\tau}^{2}} \cdot \boldsymbol{\tau}+\frac{\partial p}{\partial \boldsymbol{\tau}}=g_{\boldsymbol{\tau}},
\end{aligned}
$$

where we have set $g_{\mathbf{n}}=\mathbf{g} \cdot \mathbf{n}, g_{\boldsymbol{\tau}}=\mathbf{g} \cdot \boldsymbol{\tau}$. Averaging Eq. (3.5a) across $\gamma$ as we did for the mass balance equation we obtain:

$$
\delta \mathbf{M}_{\mathbf{n n}} U_{\mathbf{n}}+\delta \mathbf{M}_{\mathbf{n} \tau} U_{\boldsymbol{\tau}}-\left.\varepsilon \frac{\partial \mathbf{u}}{\partial \mathbf{n}} \cdot \mathbf{n}\right|_{\gamma_{2}}+\left.\varepsilon \frac{\partial \mathbf{u}}{\partial \mathbf{n}} \cdot \mathbf{n}\right|_{\gamma_{1}}-\delta \varepsilon \frac{\partial^{2} U_{\mathbf{n}}}{\partial \boldsymbol{\tau}^{2}}+\left.p\right|_{\gamma_{2}}-\left.p\right|_{\gamma_{1}}=\delta G_{\mathbf{n}},
$$


where we have set $U_{\mathbf{n}}=\frac{1}{\delta} \int_{-\delta / 2}^{\delta / 2} \mathbf{u} \cdot \mathbf{n} d \mathbf{n}$ and $G_{\mathbf{n}}=\frac{1}{\delta} \int_{-\delta / 2}^{\delta / 2} \mathbf{g} \cdot \mathbf{n} d \mathbf{n}$. Using coupling conditions (2.5b) we obtain a one-dimensional law for the flux between the two Darcy domains:

$$
\left.p_{2}\right|_{\gamma_{2}}-\left.p_{1}\right|_{\gamma_{1}}=\delta\left(G_{\mathbf{n}}-\mathbf{M}_{\mathbf{n n}} U_{\mathbf{n}}-\mathbf{M}_{\mathbf{n} \tau} U_{\boldsymbol{\tau}}+\varepsilon \frac{\partial^{2} U_{\mathbf{n}}}{\partial s^{2}}\right) .
$$

Remark 3 If the domain is fractureless (i.e. $\delta=0$ ), Eq. (3.6) expresses the continuity of pressure, i.e. the natural Darcy-Darcy coupling condition.

Analogously, by averaging Eq. (3.5b) across $\gamma$ we obtain:

$$
\delta\left(\mathbf{M}_{\boldsymbol{\tau} \mathbf{n}} U_{\mathbf{n}}+\mathbf{M}_{\boldsymbol{\tau} \boldsymbol{\tau}} U_{\boldsymbol{\tau}}\right)-\left.\varepsilon \frac{\partial \mathbf{u}}{\partial \mathbf{n}} \cdot \boldsymbol{\tau}\right|_{\gamma_{2}}+\left.\varepsilon \frac{\partial \mathbf{u}}{\partial \mathbf{n}} \cdot \boldsymbol{\tau}\right|_{\gamma_{1}}-\delta \varepsilon \frac{\partial^{2} U_{\boldsymbol{\tau}}}{\partial \boldsymbol{\tau}^{2}}+\delta \frac{\partial P}{\partial \boldsymbol{\tau}}=\delta G_{\boldsymbol{\tau}},
$$

where $P=\frac{1}{\delta} \int_{-\delta / 2}^{\delta / 2} p d \mathbf{n}$ and $G_{\boldsymbol{\tau}}=\frac{1}{\delta} \int_{-\delta / 2}^{\delta / 2} \mathbf{g} \cdot \boldsymbol{\tau} d \mathbf{n}$. Using coupling conditions (2.5) we obtain a one-dimensional balance law for the momentum on $\gamma$ :

$$
\delta\left(\mathbf{M}_{\boldsymbol{\tau} \mathbf{n}} U_{\mathbf{n}}+\mathbf{M}_{\boldsymbol{\tau} \tau} U_{\tau}-\varepsilon \frac{\partial^{2} U_{\boldsymbol{\tau}}}{\partial s^{2}}+\frac{\partial P}{\partial s}-G_{\boldsymbol{\tau}}\right)=0 .
$$

Remark 4 If the domain is fractureless, Eq. (3.7) vanishes. This is coherent with the fact that for a Darcy-Darcy coupled model only two coupling conditions are required.

Remark 5 The lines $\gamma_{1}$ and $\gamma_{2}$ are distinct and do not coincide with $\gamma$. In particular, the reduced one-dimensional model lives on the "mid-line" $\gamma$ and the geometry of the porous medium remains unaltered. Our approach is new with respect to what has usually been proposed in other works, where the two lines $\gamma_{1}$ and $\gamma_{2}$ are collapsed in order to obtain a single interface problem $[13,21,30]$. In particular, for the case of a $1 \mathrm{D}$ vessel in a $3 D$ domain, we can avoid the difficulties of treating singular solutions.

\subsection{Closure conditions}

The coupling of the Darcy equations in $\Omega_{1}$ and $\Omega_{2}$ with the reduced model for the fracture flow just derived takes place by means of interface data. Precisely, on one hand the normal velocities of the Darcy model appear as source terms in the fluid mass balance Eq. (3.3) of the fracture model; on the other, the values of the normal velocities and fluid pressure of the fracture model over the interfaces $\gamma_{i}, i=1,2$, are needed to solve the Darcy flow to impose the interface conditions on $\gamma_{i}$ in Eq. (3.2). Unfortunately, the reduced model is unable to extrapolate the fracture boundary values $p_{\mid \gamma_{i}}$ and $\mathbf{u}_{\mid \gamma_{i}} \cdot \mathbf{n}_{i}$ (referred to as FBV) from the corresponding average values $P$ and $U_{\mathbf{n}}$, so that suitable closure assumptions are needed. The idea, taken from $[19,20,36]$, is to make some a priori hypotheses on the profiles of pressure and normal velocity on a generic $s$-section of $\Omega_{f}$. More specifically, the profiles are given by interpolation of the FBV. Once the profiles are known, the average values $P, U_{\mathbf{n}}$ are easily expressed in terms of the FBV, that we can further eliminate in favour of the Darcy's 
Table 1 List of closure conditions depending on the profile associated to $P$ and $U_{\mathbf{n}}$ on a generic section of $\Omega_{f}$ and the corresponding tuning parameter $\theta$ for the closure model introduced

\begin{tabular}{llll}
\hline Profiles & & Closure conditions & $\theta$ \\
\hline$P$ & $\mathrm{C}$ & $0=\delta\left(P-\left.p_{2}\right|_{\gamma_{2}}\right)$ & NA \\
$U_{\mathbf{n}}$ & $\mathrm{C}$ & $0=\delta\left(P-\left.p_{1}\right|_{\gamma_{1}}\right)$ & \\
\hline$P$ & $\mathrm{C}$ & $-\left.2 \varepsilon \mathbf{u}_{1} \cdot \mathbf{n}_{1}\right|_{\gamma_{1}}=-2 \varepsilon U_{\mathbf{n}}+\delta\left(P-\left.p_{2}\right|_{\gamma_{2}}\right)$ & \\
$U_{\mathbf{n}}$ & $\mathrm{L}$ & $-\left.2 \varepsilon \mathbf{u}_{2} \cdot \mathbf{n}_{2}\right|_{\gamma_{2}}=+2 \varepsilon U_{\mathbf{n}}+\delta\left(P-\left.p_{1}\right|_{\gamma_{1}}\right)$ & $\frac{1}{2}$ \\
\hline$P$ & $\mathrm{~L}$ & $\left.\delta p_{1}\right|_{\gamma_{1}}-\left.4 \varepsilon \mathbf{u}_{1} \cdot \mathbf{n}_{1}\right|_{\gamma_{1}}=\delta P-4 \varepsilon U_{\mathbf{n}}+\delta\left(P-\left.p_{2}\right|_{\gamma_{2}}\right)$ & \\
$U_{\mathbf{n}}$ & $\mathrm{L}$ & $\left.\delta p_{2}\right|_{\gamma_{2}}-\left.4 \varepsilon \mathbf{u}_{2} \cdot \mathbf{n}_{2}\right|_{\gamma_{2}}=\delta P+4 \varepsilon U_{\mathbf{n}}+\delta\left(P-\left.p_{1}\right|_{\gamma_{1}}\right)$ & $\frac{2}{3}$ \\
\hline$P$ & $\mathrm{C}$ & $\left.2 \delta p_{1}\right|_{\gamma_{1}}-\left.6 \varepsilon \mathbf{u}_{1} \cdot \mathbf{n}_{1}\right|_{\gamma_{1}}=2 \delta P-6 \varepsilon U_{\mathbf{n}}+\delta\left(P-\left.p_{2}\right|_{\gamma_{2}}\right)$ & $\frac{3}{4}$ \\
$U_{\mathbf{n}}$ & $\mathrm{Q}$ & $\left.2 \delta p_{2}\right|_{\gamma_{2}}-\left.6 \varepsilon \mathbf{u}_{2} \cdot \mathbf{n}_{2}\right|_{\gamma_{2}}=2 \delta P+6 \varepsilon U_{\mathbf{n}}+\delta\left(P-\left.p_{1}\right|_{\gamma_{1}}\right)$ & \\
\hline$P$ & $\mathrm{C}$ & $\left.3 \delta p_{1}\right|_{\gamma_{1}}-\left.8 \varepsilon \mathbf{u}_{1} \cdot \mathbf{n}_{1}\right|_{\gamma_{1}}=3 \delta P-8 \varepsilon U_{\mathbf{n}}+\delta\left(P-\left.p_{2}\right|_{\gamma_{2}}\right)$ & \\
$U_{\mathbf{n}}$ & PCL & $\left.3 \delta p_{2}\right|_{\gamma_{2}}-\left.8 \varepsilon \mathbf{u}_{2} \cdot \mathbf{n}_{2}\right|_{\gamma_{2}}=3 \delta P+8 \varepsilon U_{\mathbf{n}}+\delta\left(P-\left.p_{1}\right|_{\gamma_{1}}\right)$ & \\
\hline Rel & & &
\end{tabular}

Relations are valid section by section. Labels have the following meaning: $\mathrm{C}=$ constant, $\mathrm{L}=$ linear, $\mathrm{Q}=$ quadratic, $\mathrm{PCL}=$ piecewice continuous linear (linear between $\gamma_{1}$ and $\gamma$ and between $\gamma$ and $\gamma_{2}$ )

variables $p_{i}$ and $\mathbf{u}_{i} \cdot \mathbf{n}_{i}$ using the coupling conditions (2.4), (2.5b). The interface conditions on $\gamma_{i}$ in Eq. (3.2) can thus be recast as algebraic equations coupling the Darcy's variables $p_{i}, \mathbf{u}_{i} \cdot \mathbf{n}_{i}$, with the reduced model variables $P, U_{\mathbf{n}}$. We considered different profiles: a constant or linear pressure profile, and a constant, linear, quadratic or piecewise linear normal velocity profile. Note that not all the possible combinations may be used to obtain the number of closure conditions required. The closure conditions for the combinations considered are reported in Table 1. We can see that they are equivalent, since all of them (except for the CC case) may be rewritten as:

$$
\begin{aligned}
& \left.\theta p_{1}\right|_{\gamma_{1}}-\left.\alpha \mathbf{u}_{1} \cdot \mathbf{n}_{1}\right|_{\gamma_{1}}=\theta P-\alpha U_{\mathbf{n}}+(1-\theta)\left(P-\left.p_{2}\right|_{\gamma_{2}}\right), \\
& \left.\theta p_{2}\right|_{\gamma_{2}}-\left.\alpha \mathbf{u}_{2} \cdot \mathbf{n}_{2}\right|_{\gamma_{2}}=\theta P+\alpha U_{\mathbf{n}}+(1-\theta)\left(P-\left.p_{1}\right|_{\gamma_{1}}\right),
\end{aligned}
$$

where $\alpha=\frac{2 \varepsilon}{\delta}$ and $\theta \in[0,1]$ is a nondimensional parameter depending on the profile. Table 1 also reports the different values of $\theta$ for the closure models considered.

Remark 6 The units of $\alpha$ are those of a hydraulic resistance. More precisely, $\alpha$ relates the $s$-section net balance of normal fluxes entering in (or exiting from) the fracture to the average pressure $P$ on $\gamma$ and to the pressures $p_{1}$ and $p_{2}$ in the porous media through the relation:

$$
\alpha\left(\left.\mathbf{u}_{1} \cdot \mathbf{n}_{1}\right|_{\gamma_{1}}+\left.\mathbf{u}_{2} \cdot \mathbf{n}_{2}\right|_{\gamma_{2}}\right)=\left.p_{1}\right|_{\gamma_{1}}+\left.p_{2}\right|_{\gamma_{2}}-2 P
$$

Note that if the average pressure $P$ exceeds the average of $p_{1}$ and $p_{2}$ there is a net flux exiting from the fracture at the $s$-section considered while if $P$ is lower than the 
average of $p_{1}$ and $p_{2}$ there is a net flux entering in the fracture at the $s$-section considered. From now on, we suppose that $\varepsilon \neq 0$ so that also $\alpha \neq 0$. Under this assumption, fluxes $\mathbf{u}_{1} \cdot \mathbf{n}_{1}$ and $\mathbf{u}_{2} \cdot \mathbf{n}_{2}$ may be expressed as:

$$
\begin{aligned}
& \left.\mathbf{u}_{1} \cdot \mathbf{n}_{1}\right|_{\gamma_{1}}=U_{\mathbf{n}}+\frac{\theta}{\alpha}\left(\left.p_{1}\right|_{\gamma_{1}}-P\right)+\frac{1-\theta}{\alpha}\left(\left.p_{2}\right|_{\gamma_{2}}-P\right), \\
& \left.\mathbf{u}_{2} \cdot \mathbf{n}_{2}\right|_{\gamma_{2}}=-U_{\mathbf{n}}+\frac{\theta}{\alpha}\left(\left.p_{2}\right|_{\gamma_{2}}-P\right)+\frac{1-\theta}{\alpha}\left(\left.p_{1}\right|_{\gamma_{1}}-P\right) .
\end{aligned}
$$

Note that interface fluxes $\mathbf{u}_{1} \cdot \mathbf{n}_{1}$ and $\mathbf{u}_{2} \cdot \mathbf{n}_{2}$ are obtained from the average normal flux $U_{\mathbf{n}}$ through pressure corrections depending on $\theta$.

\subsection{Weak formulation of the reduced coupled problem}

Let us start considering just the problem in the porous media, supposing for the time being that $P$ and $\alpha U_{\mathbf{n}}$ are known. We want to propose a variational formulation for the pressure in the porous media. Particularly we want to find a couple $\left(p_{1}, p_{2}\right)$ such that $(i=1,2)$ :

$$
\begin{aligned}
-\nabla \cdot\left(\mathrm{K}_{i} \nabla p_{i}\right) & =q_{i} & & \text { in } \Omega_{i}, \\
p_{i} & =\overline{p_{i}} & & \text { on } \Gamma_{i, D}, \\
\mathrm{~K}_{i} \nabla p_{i} \cdot \mathbf{n}_{i} & =h_{i} & & \text { on } \Gamma_{i, N}, \\
\left.\theta p_{1}\right|_{\gamma_{1}}-\left.\alpha \mathbf{u}_{1} \cdot \mathbf{n}_{1}\right|_{\gamma_{1}} & =\theta P-\alpha U_{\mathbf{n}}+(1-\theta)\left(P-\left.p_{2}\right|_{\gamma_{2}}\right) & & \text { on } \gamma_{1}, \\
\left.\theta p_{2}\right|_{\gamma_{2}}-\left.\alpha \mathbf{u}_{2} \cdot \mathbf{n}_{2}\right|_{\gamma_{2}} & =\theta P+\alpha U_{\mathbf{n}}+(1-\theta)\left(P-\left.p_{1}\right|_{\gamma_{1}}\right) & & \text { on } \gamma_{2} .
\end{aligned}
$$

First, we introduce the following spaces:

$$
\begin{aligned}
& V_{1}=H_{\Gamma_{1, D}}^{1}\left(\Omega_{1}\right)=\left\{v \in H^{1}\left(\Omega_{1}\right):\left.v\right|_{\Gamma_{1, D}}=0\right\}, \\
& V_{2}=H_{\Gamma_{2, D}}^{1}\left(\Omega_{2}\right)=\left\{v \in H^{1}\left(\Omega_{2}\right):\left.v\right|_{\Gamma_{2, D}}=0\right\} .
\end{aligned}
$$

and assume that the boundary data are $\bar{p}_{i} \in H^{\frac{1}{2}}\left(\Gamma_{i, D}\right), h_{i} \in H^{-\frac{1}{2}}\left(\Gamma_{i, N}\right), i=1,2$. As $\Gamma_{1, D} \neq \varnothing$ and $\Gamma_{2, D} \neq \varnothing$ by assumption, we can use the gradient seminorm in such spaces and the Poincaré inequality holds with constant $C_{P_{i}}$ (for the domain $\Omega_{i}$ ). Let us consider a vector function $\boldsymbol{v}=\left(v_{1}, v_{2}\right)$ belonging to $V=V_{1} \times V_{2}$. On the Hilbert space $V$ we use the graph norm $\|\boldsymbol{v}\|_{V}^{2}=\left\|v_{1}\right\|_{V_{1}}^{2}+\left\|v_{2}\right\|_{V_{2}}^{2}=\left\|\nabla v_{1}\right\|_{L^{2}\left(\Omega_{1}\right)}^{2}+\left\|\nabla v_{2}\right\|_{L^{2}\left(\Omega_{2}\right)}^{2}$. Multiplying the first Equation in (3.10) by a test function $r=\left(r_{1}, r_{2}\right) \in V$ and integrating over the considered domains we obtain:

$$
-\sum_{i=1,2} \int_{\Omega_{i}} \nabla \cdot\left(\mathrm{K}_{i} \nabla p_{i}\right) r_{i} d \mathbf{x}=\sum_{i=1,2} \int_{\Omega_{i}} q_{i} r_{i} d \mathbf{x}
$$


where $\boldsymbol{p}=\left(p_{1}, p_{2}\right) \in H^{1}\left(\Omega_{1}\right) \times H^{1}\left(\Omega_{2}\right)$. Integrating by parts and using the boundary conditions we have:

$$
\begin{aligned}
& \sum_{i=1,2} \int_{\Omega_{i}} \mathrm{~K}_{i} \nabla p_{i} \cdot \nabla r_{i} d \mathbf{x}-\int_{\gamma_{i}} \mathrm{~K}_{i} \nabla p_{i} \cdot \mathbf{n}_{\mathbf{i}} r_{i} d \mathbf{x} \\
& =\sum_{i=1,2} \int_{\Omega_{i}} q_{i} r_{i} d \mathbf{x}+\int_{\Gamma_{i, N}} h_{i} r_{i} d \mathbf{x} .
\end{aligned}
$$

Given that $-\mathrm{K}_{i} \nabla p_{i} \cdot \mathbf{n}_{i}=\mathbf{u}_{i} \cdot \mathbf{n}_{i}$ we can use closure conditions (3.8a) and (3.8b) in order to eliminate the normal fluxes on the interface from the (3.11). Finally we obtain:

$$
\begin{aligned}
\int_{\Omega_{1}} & \mathrm{~K}_{1} \nabla p_{1} \cdot \nabla r_{1} d \mathbf{x}+\int_{\Omega_{2}} \mathrm{~K}_{2} \nabla p_{2} \cdot \nabla r_{2} d \mathbf{x} \\
& +\frac{\theta}{\alpha} \int_{\gamma_{1}} p_{1} r_{1} d \mathbf{x}+\frac{1-\theta}{\alpha} \int_{\gamma_{1}} p_{2} r_{1} d \mathbf{x}+\frac{\theta}{\alpha} \int_{\gamma_{2}} p_{2} r_{2} d \mathbf{x}+\frac{1-\theta}{\alpha} \int_{\gamma_{2}} p_{1} r_{2} d \mathbf{x} \\
= & \int_{\Omega_{1}} q_{1} r_{1} d \mathbf{x}+\int_{\Gamma_{1, N}} h_{1} r_{1} d \mathbf{x}-\int_{\gamma_{1}} U_{\mathbf{n}} r_{1} d \mathbf{x}+\frac{1}{\alpha} \int_{\gamma_{1}} P r_{1} d \mathbf{x} \\
& +\int_{\Omega_{2}} q_{2} r_{2} d \mathbf{x}+\int_{\Gamma_{2, N}} h_{2} r_{2} d \mathbf{x}+\int_{\gamma_{2}} U_{\mathbf{n}} r_{2} d \mathbf{x}+\frac{1}{\alpha} \int_{\gamma_{2}} P r_{2} d \mathbf{x} .
\end{aligned}
$$

Let us introduce the bilinear forms $a(\cdot, \cdot), f_{2}(\cdot, \cdot)$ and $e_{2}(\cdot, \cdot)$ and the linear functional $L(\cdot)$, defined as:

$$
\begin{aligned}
a(\boldsymbol{p}, \boldsymbol{r})= & \int_{\Omega_{1}} \mathrm{~K}_{1} \nabla p_{1} \cdot \nabla r_{1} d \mathbf{x}+\int_{\Omega_{2}} \mathrm{~K}_{2} \nabla p_{2} \cdot \nabla r_{2} d \mathbf{x}+\frac{\theta}{\alpha} \int_{\gamma_{1}} p_{1} r_{1} d \mathbf{x} \\
& +\frac{1-\theta}{\alpha} \int_{\gamma_{1}} p_{2} r_{1} d \mathbf{x}+\frac{\theta}{\alpha} \int_{\gamma_{2}} p_{2} r_{2} d \mathbf{x}+\frac{1-\theta}{\alpha} \int_{\gamma_{2}} p_{1} r_{2} d \mathbf{x}, \\
L_{V}(\boldsymbol{r})= & \int_{\Omega_{1}} q_{1} r_{1} d \mathbf{x}+\int_{\Gamma_{1, N}} h_{1} r_{1} d \mathbf{x}+\int_{\Omega_{2}} q_{2} r_{2} d \mathbf{x}+\int_{\Gamma_{2, N}} h_{2} r_{2} d \mathbf{x}, \\
f_{2}(\mathbf{U}, \boldsymbol{r})= & \int_{\gamma_{2}} U_{\mathbf{n}} r_{2} d \mathbf{x}-\int_{\gamma_{1}} U_{\mathbf{n}} r_{1} d \mathbf{x}, \\
e_{2}(P, \boldsymbol{r})= & \frac{\delta}{2 \varepsilon} \int_{\gamma_{1}} P r_{1} d \mathbf{x}+\frac{\delta}{2 \varepsilon} \int_{\gamma_{2}} P r_{2} d \mathbf{x} .
\end{aligned}
$$

Owing to the surjectivity of the trace operator, introducing a lifting $R_{g} \in H^{1}\left(\Omega_{1}\right) \times$ $H^{1}\left(\Omega_{2}\right)$ of the Dirichlet data $\left(\bar{p}_{1}, \bar{p}_{2}\right)$ the problem can be rewritten as: find $p-R_{g}=$ 
$\overline{\boldsymbol{p}} \in V$ such that:

$$
a(\overline{\boldsymbol{p}}, \boldsymbol{r})=L_{V}(\boldsymbol{r})+f_{2}(\mathbf{U}, \boldsymbol{r})+e_{2}(P, \boldsymbol{r})-a\left(R_{g}, \boldsymbol{r}\right) \quad \forall \boldsymbol{r} \in V .
$$

The bilinear form $a(\cdot, \cdot)$ and the linear functionals $L_{V}(\cdot), f_{2}(\mathbf{U}, \cdot)$ and $e_{2}(P, \cdot)$ are continuous on $V$. Moreover, $a(\cdot, \cdot)$ is coercive under the assumption:

$$
\theta \geq \frac{t_{\max }}{t_{\max }+t_{\min }}
$$

In fact, we have:

$$
\begin{aligned}
a(\boldsymbol{p}, \boldsymbol{p}) \geq & \lambda_{\min }\left(K_{1}\right)\left\|p_{1}\right\|_{V}^{2}+\lambda_{\min }\left(K_{2}\right)\left\|p_{2}\right\|_{V}^{2}+\frac{\theta}{\alpha} \int_{\gamma_{1}} p_{1}^{2} d \mathbf{x} \\
& +\frac{1-\theta}{\alpha} \int_{\gamma_{1}} p_{2} p_{1} d \mathbf{x}+\frac{\theta}{\alpha} \int_{\gamma_{2}} p_{2}^{2} d \mathbf{x}+\frac{1-\theta}{\alpha} \int_{\gamma_{2}} p_{1} p_{2} d \mathbf{x} .
\end{aligned}
$$

Therefore, to prove the coercivity of the form $a(\cdot, \cdot)$, a sufficient condition is that:

$$
\frac{\theta}{\alpha} \int_{\gamma_{1}} p_{1}^{2} d \mathbf{x}+\frac{1-\theta}{\alpha} \int_{\gamma_{1}} p_{2} p_{1} d \mathbf{x}+\frac{\theta}{\alpha} \int_{\gamma_{2}} p_{2}^{2} d \mathbf{x}+\frac{1-\theta}{\alpha} \int_{\gamma_{2}} p_{1} p_{2} d \mathbf{x} \geq 0 .
$$

As

$$
\begin{aligned}
\frac{\theta}{\alpha} \int_{\gamma_{1}} p_{1}^{2} d \mathbf{x}+\frac{\theta}{\alpha} \int_{\gamma_{2}} p_{2}^{2} d \mathbf{x} & \geq \frac{\theta}{\alpha} t_{\min }\left(\left\|p_{1}\right\|_{L^{2}(\gamma)}^{2}+\left\|p_{2}\right\|_{L^{2}(\gamma)}^{2}\right), \quad \text { and } \\
\left|\frac{1-\theta}{\alpha} \int_{\gamma_{1}} p_{2} p_{1} d \mathbf{x}+\frac{1-\theta}{\alpha} \int_{\gamma_{2}} p_{1} p_{2} d \mathbf{x}\right| & \leq 2 \frac{1-\theta}{\alpha} t_{\max }\left|\int_{\gamma} p_{1} p_{2} d \mathbf{x}\right| \\
& \leq 2 \frac{1-\theta}{\alpha} t_{\max } \frac{\left\|p_{1}\right\|_{L^{2}(\gamma)}^{2}+\left\|p_{2}\right\|_{L^{2}(\gamma)}^{2}}{2},
\end{aligned}
$$

to verify the condition (3.14) it is sufficient to impose:

$$
\frac{1-\theta}{\alpha} t_{\max }\left(\left\|p_{1}\right\|_{L^{2}(\gamma)}^{2}+\left\|p_{2}\right\|_{L^{2}(\gamma)}^{2}\right) \leq \frac{\theta}{\alpha} t_{\min }\left(\left\|p_{1}\right\|_{L^{2}(\gamma)}^{2}+\left\|p_{2}\right\|_{L^{2}(\gamma)}^{2}\right),
$$

which solved for $\theta$ yields (3.13).

Note that if $\gamma$ is a straight line (3.13) becomes $\theta \geq 1 / 2$. Moreover, if the assumption (3.13) is satisfied then the solution of problem (3.12) exists and is unique and the following bound holds:

$$
\|p\|_{V} \leq \frac{1}{K_{a}}\left\|\tilde{L}_{V}\right\|_{V^{\prime}}
$$


where $K_{a}=\min \left\{\lambda_{\min }\left(K_{1}\right), \lambda_{\min }\left(K_{2}\right)\right\}$ is the coercivity constant of $a(\cdot, \cdot)$ and:

$$
\tilde{L}_{V}(\boldsymbol{r})=L_{V}(\boldsymbol{r})+f_{2}(\mathbf{U}, \boldsymbol{r})+e_{2}(P, \boldsymbol{r})-a\left(R_{g}, \boldsymbol{r}\right)
$$

Indeed these results follow from the Lax-Milgram Lemma.

Let us now focus on the reduced problem on $\gamma$, supposing that the Darcy's pressures $p_{i}$ and velocities $\mathbf{u}_{i}=-\mathrm{K}_{i} \nabla p_{i}$ in $\Omega_{1}$ and $\Omega_{2}$ are known:

$$
\begin{array}{ll}
\delta\left(\frac{\partial}{\partial s} U_{\boldsymbol{\tau}}-Q\right)=\left(\left.\mathbf{u}_{1} \cdot \mathbf{n}_{1}\right|_{\gamma_{1}}+\left.\mathbf{u}_{2} \cdot \mathbf{n}_{2}\right|_{\gamma_{2}}\right) & \text { on } \gamma, \\
\delta\left(M_{\mathbf{n n}} U_{\mathbf{n}}+M_{\mathbf{n} \tau} U_{\boldsymbol{\tau}}-\varepsilon \frac{\partial^{2} U_{\mathbf{n}}}{\partial s^{2}}\right)=\delta G_{\mathbf{n}}+\left.p_{1}\right|_{\gamma_{1}}-\left.p_{2}\right|_{\gamma_{2}} & \text { on } \gamma, \\
\delta\left(M_{\boldsymbol{\tau} \mathbf{n}} U_{\mathbf{n}}+M_{\boldsymbol{\tau} \tau} U_{\boldsymbol{\tau}}-\varepsilon \frac{\partial^{2} U_{\boldsymbol{\tau}}}{\partial s^{2}}+\frac{\partial P}{\partial s}\right)=\delta G_{\boldsymbol{\tau}} & \text { on } \gamma, \\
\quad+\text { Boundary Conditions on } \partial \gamma . &
\end{array}
$$

As $\Gamma_{f, D} \neq \varnothing$ by assumption, by averaging $\overline{\mathbf{u}}$ over that segment we obtain a Dirichlet boundary condition $\overline{\mathbf{U}}$ for $\left[U_{\mathbf{n}}, U_{\tau}\right]^{T}$. We denote with $\partial \gamma_{D}$ the boundary/ies of $\gamma$ where the averaged Dirichlet condition is assigned. Let us define the following spaces,

$$
X=\left(H_{\partial \gamma_{D}}^{1}(\gamma)\right)^{2}=\left\{\mathbf{v} \in\left(H^{1}(\gamma)\right)^{2}:\left.\mathbf{v}\right|_{\partial \gamma_{D}}=0\right\}, \quad M=L^{2}(\gamma) .
$$

Equip $X$ with the gradient seminorm (the latter is indeed a norm, thanks to the Poincare inequality), and assume the boundary data $\overline{\mathbf{U}} \in H^{\frac{1}{2}}\left(\Gamma_{f, D}\right)$. Multiplying the Eq. (3.15) times test functions $R \in M, V_{\mathbf{n}} \in H_{\partial \gamma_{D}}^{1}(\gamma)$ and $V_{\boldsymbol{\tau}} \in H_{\partial \gamma_{D}}^{1}(\gamma)$ and integrating over $\gamma$ we obtain:

$$
\begin{aligned}
\int_{\gamma} \delta & \frac{\partial}{\partial s} U_{\boldsymbol{\tau}} R d s+\int_{\gamma} \delta\left(\mathbf{M}_{\mathbf{n n}} U_{\mathbf{n}}+\mathbf{M}_{\mathbf{n} \tau} U_{\boldsymbol{\tau}}-\varepsilon \frac{\partial^{2} U_{\mathbf{n}}}{\partial s^{2}}\right) V_{\mathbf{n}} d s \\
& +\int_{\gamma} \delta\left(\mathbf{M}_{\boldsymbol{\tau} \mathbf{n}} U_{\mathbf{n}}+\mathbf{M}_{\boldsymbol{\tau} \tau} U_{\boldsymbol{\tau}}-\varepsilon \frac{\partial^{2} U_{\boldsymbol{\tau}}}{\partial s^{2}}+\frac{\partial P}{\partial s}\right) V_{\boldsymbol{\tau}} d s \\
= & \int_{\gamma}\left(\delta Q+\left.\mathbf{u}_{1} \cdot \mathbf{n}_{1}\right|_{\gamma_{1}}+\left.\mathbf{u}_{2} \cdot \mathbf{n}_{2}\right|_{\gamma_{2}}\right) R d s \\
& +\int_{\gamma}\left(\delta G_{\mathbf{n}}+\left.p_{1}\right|_{\gamma_{1}}-\left.p_{2}\right|_{\gamma_{2}}\right) V_{\mathbf{n}} d s+\int_{\gamma} \delta G_{\boldsymbol{\tau}} V_{\boldsymbol{\tau}} d s .
\end{aligned}
$$

Introducing the matrix $\mathbf{M}$ and the vectors $\mathbf{U}, \mathbf{V}$ and $\mathbf{G}$ defined as:

$$
\mathbf{M}=\left[\begin{array}{ll}
\mathrm{M}_{\mathbf{n n}} & \mathrm{M}_{\mathbf{n} \tau} \\
\mathrm{M}_{\tau \mathbf{n}} & \mathrm{M}_{\tau \tau}
\end{array}\right], \quad \mathbf{U}=\left[\begin{array}{c}
U_{\mathbf{n}} \\
U_{\tau}
\end{array}\right], \quad \mathbf{V}=\left[\begin{array}{c}
V_{\mathbf{n}} \\
V_{\tau}
\end{array}\right], \quad \mathbf{G}=\left[\begin{array}{c}
G_{\mathbf{n}} \\
G_{\tau}
\end{array}\right],
$$


the Eq. (3.16) can be rewritten as:

$$
\begin{aligned}
\int_{\gamma} \delta & \frac{\partial}{\partial s} U_{\boldsymbol{\tau}} R d s+\int_{\gamma} \delta \mathbf{M U} \cdot \mathbf{V} d s-\int_{\gamma} \delta \varepsilon \frac{\partial^{2} \mathbf{U}}{\partial s^{2}} \cdot \mathbf{V} d s+\int_{\gamma} \delta \frac{\partial P}{\partial s} V_{\boldsymbol{\tau}} d s \\
= & \int_{\gamma}\left(\delta Q+\left.\mathbf{u}_{1} \cdot \mathbf{n}_{1}\right|_{\gamma_{1}}+\left.\mathbf{u}_{2} \cdot \mathbf{n}_{2}\right|_{\gamma_{2}}\right) R d s \\
& +\int_{\gamma}\left(\left.p_{1}\right|_{\gamma_{1}}-\left.p_{2}\right|_{\gamma_{2}}\right) V_{\mathbf{n}} d s+\int_{\gamma} \delta \mathbf{G} \cdot \mathbf{V} d s .
\end{aligned}
$$

Let us introduce an averaged Neumann boundary term $\mathbf{H}=\left[H_{\mathbf{n}}, H_{\tau}\right]^{T}$ that is obtained by integrating over $\Gamma_{f, N}$ (in the case the latter is a nonempty set) the Neumann boundary data $\overline{\boldsymbol{h}} \in H^{-\frac{1}{2}}\left(\Gamma_{f, N}\right)$ as follows, where $\bar{s}$ may be 0 or $L$ :

$$
H_{\mathbf{n}}(\bar{s})=\frac{1}{\delta} \int_{\mathbf{y}(\bar{s})-(\delta / 2) \mathbf{n}}^{\mathbf{y}(\bar{s})+(\delta / 2) \mathbf{n}} \overline{\boldsymbol{h}}(\bar{s}) \cdot \mathbf{n} d \mathbf{x}, \quad H_{\boldsymbol{\tau}}(\bar{s})=\frac{1}{\delta} \int_{\mathbf{y}(\bar{s})-(\delta / 2) \mathbf{n}}^{\mathbf{y}(\bar{s})+(\delta / 2) \mathbf{n}} \overline{\boldsymbol{h}}(\bar{s}) \cdot \boldsymbol{\tau} d \mathbf{x} .
$$

By integrating by parts the Eq. (3.17), exploiting the property that $\mathbf{V}$ has null trace on $\partial \gamma_{D}$ we obtain:

$$
\begin{aligned}
\int_{\gamma} \delta & \frac{\partial}{\partial s} U_{\boldsymbol{\tau}} R d s+\int_{\gamma} \delta \mathbf{M U} \cdot \mathbf{V} d s+\int_{\gamma} \delta \varepsilon \frac{\partial \mathbf{U}}{\partial s} \cdot \frac{\partial \mathbf{V}}{\partial s} d s-\int_{\gamma} \delta \frac{\partial}{\partial s} V_{\boldsymbol{\tau}} P d s \\
= & \int_{\gamma}\left(\delta Q+\left.\mathbf{u}_{1} \cdot \mathbf{n}_{1}\right|_{\gamma_{1}}+\left.\mathbf{u}_{2} \cdot \mathbf{n}_{2}\right|_{\gamma_{2}}\right) R d s+\int_{\gamma}\left(\left.p_{1}\right|_{\gamma_{1}}-\left.p_{2}\right|_{\gamma_{2}}\right) V_{\mathbf{n}} d s \\
& +\int_{\gamma} \delta \mathbf{G} \cdot \mathbf{V} d s+\delta[\mathbf{H} \cdot \mathbf{V}]_{\partial \gamma_{N}},
\end{aligned}
$$

where we denoted $\partial \gamma_{N} \subset\{0, L\}$ the boundary of $\gamma$ where the averaged Neumann condition is assigned.

Remark 7 In Eq. (3.18) there is a term containing $\left.\mathbf{u}_{1} \cdot \mathbf{n}_{1}\right|_{\gamma_{1}}$ and $\left.\mathbf{u}_{2} \cdot \mathbf{n}_{2}\right|_{\gamma_{2}}$. As $\left.\mathbf{u}_{1} \cdot \mathbf{n}_{1}\right|_{\gamma_{1}}$ and $\left.\mathbf{u}_{2} \cdot \mathbf{n}_{2}\right|_{\gamma_{2}}$ belong to $H^{-1 / 2}\left(\gamma_{i}\right)$, their duality with a function belonging to $L^{2}(\gamma)$ is not well defined. To avoid using $a d$ hoc variational spaces we use again the closure conditions (3.8a) and (3.8b) and express the fluxes in terms of pressures and $U_{\mathbf{n}}$. 
Accordingly, using (3.8a) and (3.8b) we can rewrite Eq. (3.18) as:

$$
\begin{aligned}
\int_{\gamma} \delta & \frac{\partial}{\partial s} U_{\boldsymbol{\tau}} R d s+\int_{\gamma} \delta \mathbf{M U} \cdot \mathbf{V} d s+\int_{\gamma} \delta \varepsilon \frac{\partial \mathbf{U}}{\partial s} \cdot \frac{\partial \mathbf{V}}{\partial s} d s \\
& -\int_{\gamma} \delta \frac{\partial}{\partial s} V_{\boldsymbol{\tau}} P d s+\int_{\gamma} \frac{2}{\alpha} P R d s \\
= & \int_{\gamma} \delta Q R d s+\int_{\gamma} \frac{1}{\alpha}\left(\left.p_{1}\right|_{\gamma_{1}}+\left.p_{2}\right|_{\gamma_{2}}\right) R d s+\int_{\gamma}\left(\left.p_{1}\right|_{\gamma_{1}}-\left.p_{2}\right|_{\gamma_{2}}\right) V_{\mathbf{n}} d s \\
& +\int_{\gamma} \delta \mathbf{G} \cdot \mathbf{V} d s+\delta[\mathbf{H} \cdot \mathbf{V}]_{\partial \gamma_{N}} .
\end{aligned}
$$

Introducing the bilinear forms $c(\cdot, \cdot), b(\cdot, \cdot), d(\cdot, \cdot), f_{1}(\cdot, \cdot)$ and $e_{1}(\cdot, \cdot)$ and the linear functionals $L_{X}(\cdot)$ and $L_{M}(\cdot)$ defined as follows:

$$
\begin{aligned}
c(\mathbf{U}, \mathbf{V}) & =\delta \int_{\gamma} \mathbf{M U} \cdot \mathbf{V} d s+\delta \varepsilon \int_{\gamma} \frac{\partial \mathbf{U}}{\partial s} \cdot \frac{\partial \mathbf{V}}{\partial s} d s \\
b(\mathbf{U}, R) & =-\delta \int_{\gamma} \frac{\partial}{\partial s} U_{\tau} R d s \\
d(P, Q) & =\frac{\delta}{\varepsilon} \int_{\gamma} P R d s \\
L_{X}(\mathbf{V}) & =\delta \int_{\gamma} \mathbf{G} \cdot \mathbf{V} d s+\delta[\mathbf{H} \cdot \mathbf{V}]_{\partial \gamma_{N}}, \\
L_{M}(R) & =\delta \int_{\gamma} Q R d s \\
f_{1}(\mathbf{V}, \mathbf{p}) & =\int_{\gamma}\left(\left.p_{2}\right|_{\gamma_{2}}-\left.p_{1}\right|_{\gamma_{1}}\right) V_{\mathbf{n}} d s \\
e_{1}(R, \mathbf{p}) & =\frac{\delta}{2 \varepsilon} \int_{\gamma}\left(\left.p_{1}\right|_{\gamma_{1}}+\left.p_{2}\right|_{\gamma_{2}}\right) R d s
\end{aligned}
$$

and a lifting $\mathbf{R}_{\mathbf{g}} \in H^{1}(\gamma)$ of $\overline{\mathbf{U}}$, the problem can be rewritten in the following variational form: find $\left(\tilde{\mathbf{U}}=\mathbf{U}-\mathbf{R}_{\mathbf{g}}, P\right)$ in $X \times M$ such that

$$
\begin{array}{ll}
c(\tilde{\mathbf{U}}, \mathbf{V})+b(\mathbf{V}, P)=L_{X}(\mathbf{V})-f_{1}(\mathbf{V}, \mathbf{p})-c\left(\mathbf{R}_{\mathbf{g}}, \mathbf{V}\right) & \forall \mathbf{V} \in X \\
b(\tilde{\mathbf{U}}, R)-d(P, R)=-L_{M}(R)-e_{1}(R, \mathbf{p})-b\left(\mathbf{R}_{\mathbf{g}}, R\right) & \forall R \in M
\end{array}
$$


All the bilinear forms and the linear functionals introduced are continuous over the respective spaces, moreover the bilinear forms $c(\cdot, \cdot)$ and $d(\cdot, \cdot)$ are coercive on $X$ and $M$, respectively. Under these assumptions, the well-posedness of (3.20) follows (see, for instance, [37]).

After studying the well-posedness of the uncoupled Darcy's problem and reduced fracture model, let us focus on the full coupled model. With the previously introduced notations, the coupled model reads: find $(\mathbf{U}, P, \boldsymbol{p}) \in X \times M \times V$ such that

$$
\left\{\begin{array}{c}
c(\mathbf{U}, \mathbf{V})+b(\mathbf{V}, P)+f_{1}(\mathbf{V}, \boldsymbol{p})=L_{X}(\mathbf{V})-c\left(\mathbf{R}_{\mathbf{g}}, \mathbf{V}\right) \quad \forall \mathbf{V} \in X \\
b(\mathbf{U}, R)-d(P, R)+e_{1}(R, \boldsymbol{p})=-L_{M}(R)-b\left(\mathbf{R}_{\mathbf{g}}, R\right) \forall R \in M \\
-f_{2}(\mathbf{U}, \boldsymbol{r})-e_{2}(P, \boldsymbol{r})+a(\boldsymbol{p}, \boldsymbol{r})=L_{V}(\boldsymbol{r})-a\left(R_{g}, \boldsymbol{r}\right) \quad \forall \boldsymbol{r} \in V
\end{array}\right.
$$

To assert the well-posedness of problem (3.21) let us introduce the following result:

Theorem 1 Suppose that $X, M$ and $V$ are three Hilbert spaces normed with $\|\cdot\|_{X},\|\cdot\|_{M}$ and $\|\cdot\|_{V}$ respectively. Consider the following variational problem: find $(u, p, \phi) \in X \times M \times V$ such that:

$$
\left\{\begin{array}{rl}
c(u, v)+b(v, p)+f_{1}(v, \phi) & =L_{X}(v) \forall v \in X \\
b(u, r)-d(p, r)+e_{1}(r, \phi) & =L_{M}(r) \forall r \in M \\
-f_{2}(u, \psi)-e_{2}(p, \psi)+a(\phi, \psi) & =L_{V}(\psi) \forall \psi \in V
\end{array},\right.
$$

where: $c(\cdot, \cdot), d(\cdot, \cdot)$ and $a(\cdot, \cdot)$ are bilinear continuous and coercive forms on $X, M$, and $V$ respectively, $b(\cdot, \cdot), f_{1}(\cdot, \cdot), f_{2}(\cdot, \cdot), e_{1}(\cdot, \cdot), e_{2}(\cdot, \cdot)$ are bilinear continuous forms on $X \times M, X \times V, X \times V, M \times V$ and $M \times V$, respectively, and $L_{X}(\cdot), L_{M}(\cdot) e$ $L_{V}(\cdot)$ are linear and continuous functionals on $X, M$ and $V$, respectively. Moreover suppose that:

(i) There exists a parameter $\delta$ such that the coercivity constant $K_{c}$ and $K_{d}$ of the bilinear forms $c(\cdot, \cdot)$ and $d(\cdot, \cdot)$ can be written as $K_{c}=\delta C$ and $K_{d}=\delta D$ where $C$ and $D$ are positive constants.

(ii) There exist two functions $F(\delta)$ and $E(\delta)$ such that:

$$
\begin{aligned}
\left|f_{1}(v, \psi)-f_{2}(v, \psi)\right| & \leq \delta F(\delta)\|v\|_{X}\|\psi\|_{V} \quad \forall v \in X, \forall \psi \in V, \\
\left|e_{1}(r, \psi)+e_{2}(r, \psi)\right| & \leq \delta E(\delta)\|r\|_{M}\|\psi\|_{V} \quad \forall r \in M, \forall \psi \in V .
\end{aligned}
$$

(iii) There exist $\bar{\delta}$ and two constants $E_{\max }, F_{\max }$ such that, if $0 \leq \delta \leq \bar{\delta}$ :

$$
0 \leq E(\delta) \leq E_{\max }, \quad 0 \leq F(\delta) \leq F_{\max }
$$

Then there exists $\delta^{*}<\bar{\delta}$ such that $\forall \delta: 0<\delta<\delta^{*}$ the solution of the Problem (3.22) exists and is unique. Moreover $\delta^{*}$ can be chosen as:

$$
\delta^{*}=\min \left\{\frac{1}{3} \frac{K_{a} C D}{C E_{\max }^{2}+D F_{\max }^{2}}, \bar{\delta}\right\}
$$


Besides, under these assumptions the following estimates hold:

$$
\|\phi\|_{V},\|p\|_{M},\|u\|_{X} \leq \frac{1}{K_{S}(\delta)}\left(C_{L_{X}}+C_{L_{X}}+C_{L_{V}}\right)
$$

where $C_{L_{X}}, C_{L_{M}}$ and $C_{L_{V}}$ are the continuity constants of the functionals $L_{X}, L_{M}$ and $L_{V}$, respectively, $K_{S}(\delta)=\delta K_{s}^{*}$, and $K_{s}^{*}$ is a constant depending on $C, D, E_{\max }$ and $F_{\max }$.

Proof Let us introduce the product space $H=X \times M \times V$, so that $\mathbf{h}=\left(h_{X}, h_{M}, h_{V}\right)$, and define $\|\mathbf{h}\|_{H}^{2}=\left\|h_{X}\right\|_{X}^{2}+\left\|h_{M}\right\|_{M}^{2}+\left\|h_{V}\right\|_{V}^{2}$.

Consider the bilinear form $s(\cdot, \cdot): H \times H \rightarrow \mathbb{R}$ and the linear functional $L_{H}(\cdot): H \rightarrow \mathbb{R}$ defined as:

$$
\begin{aligned}
s(\mathbf{h}, \mathbf{z})= & c\left(h_{X}, z_{X}\right)+b\left(z_{X}, h_{M}\right)+f_{1}\left(z_{X}, h_{V}\right)+b\left(h_{X}, z_{M}\right)-d\left(h_{M}, z_{M}\right) \\
& +e_{1}\left(z_{M}, h_{V}\right)-f_{2}\left(h_{X}, z_{V}\right)-e_{2}\left(h_{M}, z_{V}\right)+a\left(h_{V}, z_{V}\right) \\
L_{H}(\mathbf{z})= & L_{X}\left(z_{X}\right)+L_{M}\left(z_{M}\right)+L_{V}\left(z_{V}\right) .
\end{aligned}
$$

Problem (3.22) is equivalent to finding $\mathbf{h} \in H$ such that:

$$
s(\mathbf{h}, \mathbf{z})=L_{H}(\mathbf{z}) \quad \forall \mathbf{z} \in H .
$$

Let us show that $s(\cdot, \cdot)$ satisfies the hypotheses of the Banach-Nečas-Babuška Theorem $^{2}$ [18]. $s(\cdot, \cdot)$ and $L_{H}(\cdot)$ are continuous. We will show that, for $\delta$ small enough, there exists $K_{s}=\delta K_{s}^{*}\left(C, D, E_{\max }, F_{\max }\right)>0$ such that:

$$
\sup _{\mathbf{z} \in H, \mathbf{z} \neq \mathbf{0}} \frac{s(\mathbf{h}, \mathbf{z})}{\|\mathbf{z}\|_{H}} \geq K_{s}\|\mathbf{h}\|_{H} .
$$

Choose $\mathbf{z}=\left(h_{X},-h_{M}, h_{V}\right)$; correspondingly $\|\mathbf{z}\|_{H}=\|\mathbf{h}\|_{H}$, and:

$$
\begin{aligned}
s(\mathbf{h}, \mathbf{z}) \geq & \delta C\left\|h_{X}\right\|_{X}^{2}+\delta D\left\|h_{M}\right\|_{M}^{2}+\vartheta K_{a}\left\|h_{V}\right\|_{V}^{2}+(1-\vartheta) K_{a}\left\|h_{V}\right\|_{V}^{2} \\
& -\delta F(\delta)\left\|h_{X}\right\|_{X}\left\|h_{V}\right\|_{V}-\delta E(\delta)\left\|h_{M}\right\|_{M}\left\|h_{V}\right\|_{V},
\end{aligned}
$$

for any parameter $\vartheta \in(0,1)$, were $K_{a}$ is the coercivity constant of $a(\cdot, \cdot)$. We want to find under which condition there exist two positive constants $\alpha_{X}$ and $\alpha_{M}$ such that:

$$
\begin{aligned}
I_{X} & =\delta C\left\|h_{X}\right\|_{X}^{2}-\delta F(\delta)\left\|h_{X}\right\|_{X}\left\|h_{V}\right\|_{V}+\vartheta K_{a}\left\|h_{V}\right\|_{V}^{2} \\
& \geq \delta \alpha_{X}\left(\left\|h_{X}\right\|_{X}^{2}+\left\|h_{V}\right\|_{V}^{2}\right), \\
I_{M} & =\delta D\left\|h_{M}\right\|_{M}^{2}-\delta E(\delta)\left\|h_{M}\right\|_{M}\left\|h_{V}\right\|_{V}+(1-\vartheta) K_{a}\left\|h_{V}\right\|_{V}^{2} \\
& \geq \delta \alpha_{M}\left(\left\|h_{M}\right\|_{M}^{2}+\left\|h_{V}\right\|_{V}^{2}\right) .
\end{aligned}
$$

In fact, in that case (3.27) follows with $K_{s}=\delta K_{s}^{*}, K_{s}^{*}=\min \left\{\alpha_{X}, \alpha_{M}\right\}$.

$\overline{2}$ From now on we will refer to it as the BNB Theorem. 
Consider first $I_{X}$. For any $\epsilon>0$, by Young's inequality we have

$$
I_{X} \geq \delta\left[(C-F(\delta) \epsilon)\left\|h_{X}\right\|_{X}^{2}+\left(\vartheta K_{a} \frac{1}{\delta}-F(\delta) \frac{1}{\epsilon}\right)\left\|h_{V}\right\|_{V}^{2}\right] \text {. }
$$

We then choose $\epsilon=\frac{1}{2} \frac{C}{F(\delta)}$ and get

$$
\begin{aligned}
I_{X} & \geq \delta\left[\frac{1}{2} C\left\|h_{X}\right\|_{X}^{2}+\left(\vartheta K_{a} \frac{1}{\delta}-2 \frac{F(\delta)^{2}}{C}\right)\left\|h_{V}\right\|_{V}^{2}\right] \\
& \geq \delta C\left[\frac{1}{2}\left\|h_{X}\right\|_{X}^{2}+\frac{F_{\max }^{2}}{C^{2}}\left\|h_{V}\right\|_{V}^{2}\right] \text { if } \delta<\min \left\{\bar{\delta}, \vartheta \frac{1}{3} \frac{K_{a} C}{F_{\max }^{2}}\right\},
\end{aligned}
$$

i.e. $\alpha_{X}=\min \left\{\frac{C}{2}, \frac{F_{\max }^{2}}{C}\right\}$. Analogously, we get

$$
I_{M} \geq \delta D\left[\frac{1}{2}\left\|h_{M}\right\|_{M}^{2}+\frac{E_{\max }^{2}}{D^{2}}\left\|h_{V}\right\|_{V}^{2}\right] \text { if } \delta<\min \left\{\bar{\delta},(1-\vartheta) \frac{1}{3} \frac{K_{a} D}{E_{\max }^{2}}\right\}
$$

i.e. $\alpha_{M}=\min \left\{\frac{D}{2}, \frac{E_{\max }^{2}}{D}\right\}$. From (3.29) and (3.30), choosing $\vartheta$ such that

$$
\vartheta \frac{1}{3} \frac{K_{a} C}{F_{\max }^{2}}=(1-\vartheta) \frac{1}{3} \frac{K_{a} D}{E_{\max }^{2}}=\frac{1}{3} \frac{K_{a} C D}{C E_{\max }^{2}+D F_{\max }^{2}}=\widehat{\delta},
$$

we have that, for all $0<\delta<\delta^{*}=\min \{\bar{\delta}, \widehat{\delta}\}$,

$$
K_{s}=\delta K_{s}^{*}=\delta \min \left\{\frac{C}{2}, \frac{D}{2}, \frac{F_{\max }^{2}}{C}, \frac{E_{\max }^{2}}{D}\right\}>0,
$$

and condition (3.27) follows. The condition:

$$
\sup _{\mathbf{h} \in H} s(\mathbf{h}, \mathbf{z})>0 \quad \forall \mathbf{z} \neq \mathbf{0},
$$

can be proved by proceeding in the same way. As all the hypotheses of the BNB Theorem are satisfied the existence and uniqueness of the solution of problem (3.26) follows and consequently the solution of problem (3.22) exists and is unique. BNB Theorem also provides the a priori estimate (3.24), since

$$
\|\mathbf{h}\|_{H} \leq \frac{1}{K_{S}}\left(C_{L_{X}}+C_{L_{M}}+C_{L_{V}}\right) .
$$

Note that this result states that problem (3.20) is well posed for $\delta$ small enough, but at the same time the stability constant $K_{s}$ degenerates for $\delta \rightarrow 0$ (linearly). Precisely, Eq. (3.31) and (3.32) quantify on one hand "how small" $\delta$ has to be (see the expression of $\widehat{\delta}$ ), and on the other hand the dependance of $K_{S}$ on $\delta, C, D, E_{\max }, F_{\max }$. This is 
of particular interest when considering the Galerkin approximation of (3.20), since $\delta$ enters the error estimate (see Sect. 5) via $K_{S}$.

The boundedness assumption on $E(\delta)$ and $F(\delta)$ in Theorem 1 can be relaxed, as stated below.

Remark 8 Theorem 1 still holds if the hypothesis (iii) is replaced by the following one:

(iii-d) There exists $\bar{\delta}$ and four constants $m_{f}>0, m_{e}>0, r_{f}<1 / 2$ and $r_{e}<1 / 2$ such that, if $0 \leq \delta \leq \bar{\delta}$ :

$$
E(\delta) \leq m_{e} \delta^{-r_{e}}, \quad F(\delta) \leq m_{f} \delta^{-r_{f}} .
$$

In this case $\delta^{*}=\delta^{*}\left(K_{a}, C, D, m_{e}, m_{f}, r_{e}, r_{f}\right), K_{s}^{*}=K_{s}^{*}\left(C, D, m_{e}, m_{f}\right)$.

Remark 8 follows using exactly the same arguments of Theorem 1. In particular, (3.29) can be written as $I_{X} \geq \delta C\left[\frac{1}{2}\left\|h_{X}\right\|_{X}^{2}+\frac{m_{f}^{2}}{C^{2}}\left\|h_{V}\right\|_{V}^{2}\right]$ provided that $\delta<\min \{\bar{\delta}$, $\left.\left(\frac{\vartheta K_{a} C}{3 m_{f}^{2}}\right)^{\frac{1}{1-2 r_{f}}}\right\}$, and a similar estimate can replace Eq. (3.30).

Finally, let us show that problem 3.21 satisfies the assumptions of Theorem 1 if we choose the parameter $\delta$ to be the width of the fracture. Then the following result holds:

Theorem 2 There exists a positive number $\delta^{*}$ such that if $0<\delta<\delta^{*}$ the solution of Problem 3.21 exists and is unique.

Proof Problem (3.21) has the same form of (3.22) and it is easy to show that $c(\cdot, \cdot)$, $d(\cdot, \cdot)$ and $a(\cdot, \cdot)$ are bilinear continuous and coercive forms on $X, M$, and $V$ respectively, $b(\cdot, \cdot), f_{1}(\cdot, \cdot), f_{2}(\cdot, \cdot), e_{1}(\cdot, \cdot), e_{2}(\cdot, \cdot)$ are bilinear continuous forms on $X \times M$, $X \times V, X \times V, M \times V$ and $M \times V$, respectively, and $L_{X}(\cdot), L_{M}(\cdot)$ and $L_{V}(\cdot)$ are linear and continuous functionals on $X, M$ and $V$, respectively. Let us prove that the assumptions of Theorem 1 are satisfied. The coercivity constants $K_{c}=\varepsilon \delta$ and $K_{d}=\frac{1}{\varepsilon} \delta$ are as required by the first hypothesis of Theorem 1 (with $C=$ $\left.\varepsilon, D=\frac{1}{\varepsilon}\right)$. Let us identify the two functions $F(\delta)$ and $E(\delta)$. For what concerns $F(\delta)$, let $\Delta_{F}=\left|f_{1}(\mathbf{V}, \boldsymbol{r})-f_{2}(\mathbf{V}, \boldsymbol{r})\right|$; we then have

$$
\begin{aligned}
\Delta_{F} & =\left|\int_{\gamma}\left(\left.r_{2}\right|_{\gamma_{2}}-\left.r_{1}\right|_{\gamma_{1}}\right) V_{\mathbf{n}} d \mathbf{x}-\int_{\gamma_{2}} r_{2}\right|_{\gamma_{2}} V_{\mathbf{n}} d \mathbf{x}+\left.\int_{\gamma_{1}} r_{1}\right|_{\gamma_{1}} V_{\mathbf{n}} d \mathbf{x} \mid \\
& =\left|\int_{\gamma}\left(\left.r_{2}\right|_{\gamma_{2}}-\left.r_{1}\right|_{\gamma_{1}}\right) V_{\mathbf{n}} d \mathbf{x}-\int_{\gamma} r_{2}\right|_{\gamma_{2}} V_{\mathbf{n}} \frac{r \pm \delta / 2}{r} d \mathbf{x}+\left.\int_{\gamma} r_{1}\right|_{\gamma_{1}} V_{\mathbf{n}} \frac{r \mp \delta / 2}{r} d \mathbf{x} \mid \\
& =\left|\int_{\gamma} r_{2}\right|_{\gamma_{2}} V_{\mathbf{n}}\left(1-\frac{r \pm \delta / 2}{r}\right) d \mathbf{x}-\left.\int_{\gamma} r_{1}\right|_{\gamma_{1}} V_{\mathbf{n}}\left(1-\frac{r \mp \delta / 2}{r}\right) d \mathbf{x} \mid
\end{aligned}
$$




$$
\begin{aligned}
& =\left|\int_{\gamma} r_{2}\right|_{\gamma_{2}} V_{\mathbf{n}} \frac{\mp \delta / 2}{r} d \mathbf{x}-\left.\int_{\gamma} r_{1}\right|_{\gamma_{1}} V_{\mathbf{n}} \frac{ \pm \delta / 2}{r} d \mathbf{x} \mid \\
& \leq \frac{\delta / 2}{r_{\min }}\left(\left.\left|\int_{\gamma} r_{2}\right|_{\gamma_{2}} V_{\mathbf{n}} d \mathbf{x}|+| \int_{\gamma} r_{1}\right|_{\gamma_{1}} V_{\mathbf{n}} d \mathbf{x} \mid\right) \\
& \leq \frac{\delta / 2}{r_{\min }}\left(\left\|\left.r_{2}\right|_{\gamma_{2}}\right\|_{L^{2}(\gamma)}+\left\|\left.r_{1}\right|_{\gamma_{1}}\right\|_{L^{2}(\gamma)}\right)\left\|V_{\mathbf{n}}\right\|_{L^{2}(\gamma)} \\
& \leq \frac{\delta / 2}{r_{\min }} \sqrt{t_{\max }}\left(\left\|\left.r_{2}\right|_{\gamma_{2}}\right\|_{L^{2}\left(\gamma_{2}\right)}+\left\|\left.r_{1}\right|_{\gamma_{1}}\right\|_{L^{2}\left(\gamma_{1}\right)}\right)\left\|V_{\mathbf{n}}\right\|_{L^{2}(\gamma)} \\
& \leq \delta \frac{1}{2} \frac{\left(r_{\min }+\delta / 2\right)^{1 / 2}}{r_{\min }\left(r_{\min }-\delta / 2\right)^{1 / 2}}\left(C_{T_{1}}+C_{T_{2}}\right) C_{P_{f}}\|\boldsymbol{r}\|_{V}\|V\|_{X},
\end{aligned}
$$

where $C_{T_{1}}$ and $C_{T_{2}}$ are trace constants and $C_{P_{f}}$ is the Poincare constant. Therefore we can define $F(\delta)$ as:

$$
F(\delta)=\frac{1}{2} \frac{\left(r_{\min }+\delta / 2\right)^{1 / 2}}{r_{\min }\left(r_{\min }-\delta / 2\right)^{1 / 2}}\left(C_{T_{1}}+C_{T_{2}}\right) C_{P_{f}} .
$$

For what concerns $E(\delta)$, let $\Delta_{E}=\left|e_{1}(R, \boldsymbol{r})+e_{2}(R, \boldsymbol{r})\right|$; we have

$$
\begin{aligned}
\Delta_{E}= & \left|\frac{\delta}{2 \varepsilon} \int_{\gamma}\left(\left.r_{2}\right|_{\gamma_{2}}+\left.r_{1}\right|_{\gamma_{1}}\right) P d \mathbf{x}+\frac{\delta}{2 \varepsilon} \int_{\gamma_{2}} r_{2}\right|_{\gamma_{2}} P d \mathbf{x}+\left.\frac{\delta}{2 \varepsilon} \int_{\gamma_{1}} r_{1}\right|_{\gamma_{1}} P d \mathbf{x} \mid \\
= & \frac{\delta}{2 \varepsilon} \int_{\gamma}\left(\left.r_{2}\right|_{\gamma_{2}}+\left.r_{1}\right|_{\gamma_{1}}\right) P d \mathbf{x}+\left.\frac{\delta}{2 \varepsilon} \int_{\gamma} r_{2}\right|_{\gamma_{2}} P \frac{r \pm \delta / 2}{r} d \mathbf{x} \\
& +\left.\frac{\delta}{2 \varepsilon} \int_{\gamma} r_{1}\right|_{\gamma_{1}} P \frac{r \mp \delta / 2}{r} d \mathbf{x} \mid \\
\leq & \frac{\delta}{2 \varepsilon} \frac{2 r_{\min }+\delta / 2}{r_{\min }} \int_{\gamma}\left|\left(\left.r_{2}\right|_{\gamma_{2}}+\left.r_{1}\right|_{\gamma_{1}}\right) P\right| d \mathbf{x} \\
\leq & \frac{\delta}{2 \varepsilon} \frac{2 r_{\min }+\delta / 2}{r_{\min }}\left(\left\|\left.r_{2}\right|_{\gamma_{2}}\right\|_{L^{2}(\gamma)}+\left\|\left.r_{1}\right|_{\gamma_{1}}\right\|_{L^{2}(\gamma)}\right)\|P\|_{M} \\
\leq & \frac{\delta}{2 \varepsilon} \sqrt{t_{\max }} \frac{2 r_{\min }+\delta / 2}{r_{\min }}\left(\left\|\left.r_{2}\right|_{\gamma_{2}}\right\|_{L^{2}\left(\gamma_{2}\right)}+\left\|\left.r_{1}\right|_{\gamma_{1}}\right\|_{L^{2}\left(\gamma_{1}\right)}\right)\|P\|_{M} \\
\leq & \frac{\delta}{2 \varepsilon} \sqrt{t_{\max }} \frac{2 r_{\min }+\delta / 2}{r_{\min }}\left(C_{T_{1}}+C_{T_{2}}\right)\|\boldsymbol{r}\|_{V}\|P\|_{M} \\
\leq & \frac{1}{2 \varepsilon} \frac{\left(2 r_{\min }+\delta / 2\right)\left(r_{\min }+\delta / 2\right)^{1 / 2}}{r_{\min }\left(r_{\min }-\delta / 2\right)^{1 / 2}}\left(C_{T_{1}}+C_{T_{2}}\right)\|\boldsymbol{r}\|_{V}\|P\|_{M},
\end{aligned}
$$


therefore we can define $E(\delta)$ as:

$$
E(\delta)=\frac{1}{2 \varepsilon} \frac{\left(r_{\min }+\delta / 2\right)^{1 / 2}\left(2 r_{\min }+\delta / 2\right)}{r_{\min }\left(r_{\min }-\delta / 2\right)^{1 / 2}}\left(C_{T_{1}}+C_{T_{2}}\right)
$$

The estimates for $F(\delta)$ and $E(\delta)$ are clearly uniformly bounded w.r.t. $\delta$ for $\delta$ small. In fact, as we have $\delta / 2<\bar{r} \leq r_{\min }$, for any number $\tilde{r}<r_{\min }$, we have that for all $0<\delta<2 \tilde{r}$ the following inequalities hold:

$$
\begin{aligned}
& 0 \leq F(\delta) \leq \frac{1}{2} \frac{\left(r_{\min }+\tilde{r}\right)^{1 / 2}}{r_{\min }\left(r_{\min }-\tilde{r}\right)^{1 / 2}}\left(C_{T_{1}}+C_{T_{2}}\right) C_{P_{f}}, \\
& 0 \leq E(\delta) \leq \frac{1}{2 \varepsilon} \frac{\left(r_{\min }+\tilde{r}\right)^{1 / 2}\left(2 r_{\min }+\tilde{r}\right)}{r_{\min }\left(r_{\min }-\tilde{r}\right)^{1 / 2}}\left(C_{T_{1}}+C_{T_{2}}\right),
\end{aligned}
$$

i.e. the third hypothesis of Theorem 1 is satisfied. For instance, we can take $\tilde{r}=r_{\min } / 2$ so that $F_{\max }=\frac{\sqrt{3}}{2 r_{\min }}\left(C_{T_{1}}+C_{T_{2}}\right) C_{P_{f}}, E_{\max }=\frac{5 \sqrt{3}}{4 \varepsilon}\left(C_{T_{1}}+C_{T_{2}}\right)$ and choose $\delta^{*}$ as:

$$
\delta^{*}=\min \left\{r_{\min }, \widehat{\delta}\right\}, \quad \widehat{\delta}=\frac{16 K_{a} \varepsilon r_{\min }^{2}}{9\left(C_{T_{1}}+C_{T_{2}}\right)^{2}\left(25 r_{\min }^{2}+4 C_{P_{f}}^{2}\right)}
$$

As a result, we see that a small $\delta^{*}$ can be due to degeneration of $\varepsilon$, of the coercivity constant $K_{a}=\min \left\{\lambda_{\min }\left(K_{1}\right), \lambda_{\min }\left(K_{2}\right)\right\}$, or to high curvatures $r_{\min }^{-1}$.

\section{Extension to the case of a fracture or a vessel in a three-dimensional domain}

In this section we indicate how the model detailed in Sect. 3 can be extended to the case of a fracture or a vessel in a three-dimensional porous medium (see Fig. 1). The substantial difference with respect to the $2 \mathrm{D}$ case is that in every point of the mean surface of the fracture or of the mean line of the vessel, the intrinsic reference system is constituted by three orthonormal unit vectors instead of two.

The case of a fracture in a three-dimensional domain. In this case there are two tangential and one normal unit vectors. The procedure highlighted in Sect. 2 can be carried out without too many difficulties, the derivation of the reduced model is straightforward and the same closure conditions used in Sect. 3 may be used to couple the reduced model with the Darcy problem in the porous medium. However in order to obtain a unique equation on the whole mean surface of the fracture a suitable reference system should be introduced on such surface. The well-posedness of the problem under the assumption that the width of the fracture is small enough may be proved by either Theorem 1 or one of its variants. The structure of the variational problem is in fact the same as (3.21) but the variational spaces of the reduced models are defined on a bi-dimensional geometry. For a detailed description of this case refer to [28]. 
The case of a vessel in a three-dimensional domain. In this case there are two normal and one tangential unit vectors. This time a suitable reference system must be chosen at every section of the vessel so that the two normal unit vectors vary with continuity with respect to the arc length. The averaging operations may be carried out straightforwardly, however (2.5a) and (2.5b) have to be rewritten in the following vector form:

$$
\begin{aligned}
p_{D} \hat{\mathbf{n}} & =p_{S} \hat{\mathbf{n}}-\varepsilon \mathrm{N} \frac{\partial \mathbf{u}}{\partial \hat{\mathbf{n}}} & & \text { on } \Gamma_{D B}, \\
0 & =-\varepsilon \mathrm{T} \frac{\partial \mathbf{u}}{\partial \hat{\mathbf{n}}} & & \text { on } \Gamma_{D B} .
\end{aligned}
$$

Here $\hat{\mathbf{n}}=[\cos (\beta), \sin (\beta)]$ represents the normal external unit vector of the vessel with respect to the orthonormal bi-dimensional reference system given by the two normal unit vectors; $\beta$ is the angular coordinate in a bi-dimensional polar coordinate system in which $\mathbf{n}_{1}$ is the polar axis. Beside this, the following revised closure condition must be used instead of (3.8a) and (3.8b):

$$
\begin{aligned}
\theta p(\beta)-\alpha(\mathbf{u} \cdot \mathbf{n})(\beta)= & \theta P+\alpha U_{\mathbf{n}_{1}} \cos \beta+\alpha U_{\mathbf{n}_{2}} \sin \beta \\
& +(1-\theta)(P-p(\beta+\pi)),
\end{aligned}
$$

where $U_{\mathbf{n}_{1}}$ and $U_{\mathbf{n}_{2}}$ represent the projection of the average flux on the two normal unit vectors and $f(\beta)$ represents the value of a generic function $f$ on the boundary of the considered section at angular coordinate $\beta$. The weak formulation of the coupled problem can be recast in the form (3.22), where the parameter $\delta$ is now the vessel cross-sectional area. The well-posedness for small $\delta$ is obtained owing to Remark 8 with $r_{e}=r_{f}=\frac{1}{4}$. For a detailed description of this case we refer to [28].

\section{Numerical approximation of the coupled problem}

In this section we briefly address the numerical solution of the reduced model problem proposed in this paper. We consider the standard Galerkin finite element approximation of the coupled problem (3.21), and introduce the discrete subspaces $X_{h} \subset X$ for the velocity in the fracture, $M_{h} \subset M$ for the fracture pressure, and $V_{h} \subset V$ for the Darcy pressures. Recall the definition of the bilinear form $s(\cdot, \cdot)$ and linear form $L_{H}(\cdot)$ from (3.25), let $H_{h}=X_{h} \times M_{h} \times V_{h} \subset H$ and consider the discrete problem of finding $\mathbf{h}_{h} \in H_{h}$ such that

$$
s\left(\mathbf{h}_{h}, \mathbf{z}_{h}\right)=L_{H}\left(\mathbf{z}_{h}\right) \quad \forall \mathbf{z}_{h} \in H_{h} .
$$

Theorem 3 If $0<\delta<\delta^{*}$, problem (5.1) has a unique solution, satisfying

$$
\left\|\mathbf{h}-\mathbf{h}_{h}\right\|_{H} \leq C \frac{1}{\delta} \inf _{\mathbf{z}_{h} \in H_{h}}\left\|\mathbf{h}-\mathbf{z}_{h}\right\|_{H}
$$

As a consequence, using standard $\mathbb{P}_{k}$ finite elements of degree $k \geq 1$ for the spaces $X_{h}, V_{h}$, and standard $\mathbb{P}_{k-1}$ elements for the space $M_{h}$, and assuming that the solution 
$\mathbf{h}=(\mathbf{U}, P, \boldsymbol{p}) \in H^{k+1}\left(\gamma_{f}\right) \times H^{k}\left(\gamma_{f}\right) \times H^{k+1}\left(\Omega_{1,2}\right)$, we have

$$
\left\|\mathbf{h}-\mathbf{h}_{h}\right\|_{H} \leq C \frac{h^{k}}{\delta}\left(|\mathbf{U}|_{H^{k+1}(\gamma)}^{2}+|P|_{H^{k}(\gamma)}^{2}+\sum_{i=1,2}\left|p_{i}\right|_{H^{k+1}\left(\Omega_{i}\right)}^{2}\right)^{\frac{1}{2}} .
$$

Proof For any $\mathbf{g}=\left(g_{X}, g_{M}, g_{V}\right) \in H$, denote $\widetilde{\mathbf{g}}=\left(g_{X},-g_{M}, g_{V}\right)$. Proceeding as in the proof of Theorem 1 , for $0<\delta<\delta^{*}$ and $\forall \mathbf{z}_{h} \in H_{h}$, we have $s\left(\mathbf{z}_{h}, \widetilde{\mathbf{z}}_{h}\right) \geq$ $K_{S}(\delta)\left\|\mathbf{z}_{h}\right\|_{H}^{2},\left\|\widetilde{\mathbf{z}}_{h}\right\|_{H}=\left\|\mathbf{z}_{h}\right\|_{H}$. Hence, the discrete solution exists and is unique. Similarly, for $\mathbf{e}=\mathbf{h}-\mathbf{h}_{h}$ and any $\mathbf{z}_{h} \in H_{h}$, owing to the Galerkin orthogonality we have

$$
K_{S}(\delta)\|\mathbf{e}\|_{H}^{2} \leq s(\mathbf{e}, \widetilde{\mathbf{e}})=s\left(\mathbf{e}, \widetilde{\mathbf{h}}-\widetilde{\mathbf{z}}_{h}\right) \leq C_{S}\|\mathbf{e}\|_{H}\left\|\mathbf{h}-\mathbf{z}_{h}\right\|_{H}
$$

where $C_{s}$ is the continuity constant of $s$. Owing to the estimate $K_{s}(\delta)=\delta K_{s}^{*}$ of Theorem 1, Eq. (5.2) follows. Equation (5.3) follows as well using standard interpolation estimates observing that $\|\mathbf{g}\|_{H}^{2}=\left\|g_{X}\right\|_{H^{1}(\gamma)}^{2}+\left\|g_{M}\right\|_{L^{2}(\gamma)}^{2}+\left\|g_{V}\right\|_{H^{1}\left(\Omega_{1}\right) \times H^{1}\left(\Omega_{2}\right)}^{2}$.

We observe that, thanks to the estimate of the stability constant $K_{S}(\delta)$ provided in Theorem 1, we can quantify the dependence of the error estimate on $\delta$. We also point out that, since every triple of finite element spaces $X_{h} \times M_{h} \times V_{h}$ is (inf-sup) stable, other choices of finite element different from those mentioned in Theorem 3 are possible. For instance, we could opt for the cheaper $\mathbb{P}^{1} \times \mathbb{P}^{1} \times \mathbb{P}^{1}$ triple instead of the $\mathbb{P}^{1} \times \mathbb{P}^{0} \times \mathbb{P}^{1}(k=1)$ retaining convergence of order 1 under the same regularity assumptions. In fact, all the numerical computations of the next section were performed using equal order (linear) continuous finite elements.

Concerning the numerical solution of such of the linear system (5.1), due to the partitioned structure of the system matrix the use of preconditioned methods for the Schur complement (see for example $[9,10,17,22,32,35,38]$ ) may be of interest. In particular, diagonal or triangular preconditioners may lead to solve separately a Poisson problem in the porous medium and a Stokes problem in the fracture. For both problems a wide variety of highly effective numerical methods and preconditioners are available (see for example $[4,6,12,27,29,31,33,40,43]$ for the Darcy problem or $[1,8,11,23,34,42,44]$ for the Stokes/Brinkman problem).

\section{Extension to a network of fractures}

The multiscale strategy presented in this work can be easily extended to networks. For the sake of simplicity, in this section we will describe the modeling of a simple network constituted by one bifurcating fracture. Consider three domains $\Omega_{i}$ separated by a $Y$-shaped region $\Omega_{f}$ as in Fig. 3, where $i \in I=\{0,1,2\}$. We shall use modular arithmetics in $I$, for instance $i+1=0$ for $i=2$. Denote by $\gamma^{i+2}$ the one-dimensional centerline of the branch between $\Omega_{i}$ and $\Omega_{i+1}$, let $\gamma=\cup_{i \in I} \gamma^{i}$, and let $\gamma_{i}$ denote the interface between $\Omega_{i}$ and $\Omega_{f}$. 


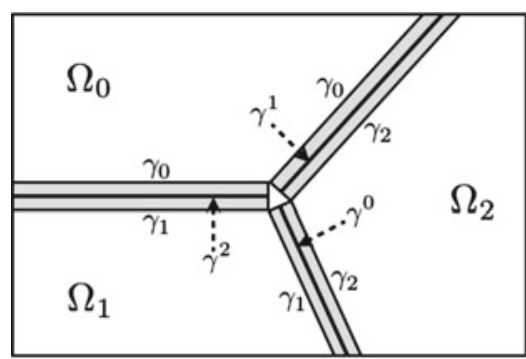

(a)

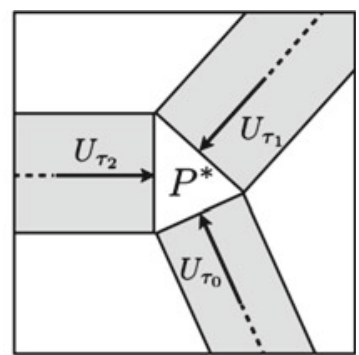

(b)

Fig. 3 a A branching channel $\Omega_{f}$ (in gray) with three exterior subdomains $\Omega_{i}$. b Magnified view of the bifurcation

As in Sect. 3, we will use local coordinates $s, \xi$ in $\Omega_{f}$ around each branch $\gamma^{i}$. The domain $\Omega_{f}$ is then covered by a global atlas by using a suitable partition of unity. Using local coordinates, we can easily construct continuous mappings $f \in L^{2}\left(\gamma_{i+1}\right) \times L^{2}\left(\gamma_{i+2}\right) \mapsto \tilde{f} \in L^{2}\left(\gamma_{i}\right)$ and $f \in L^{2}(\gamma) \mapsto \hat{f} \in L^{2}\left(\gamma_{i}\right)$, which are the counterparts of the extension maps described in Sect. 3.1, and provide respectively values taken on the "opposite" channel—porous medium interface, and on the centerline of the channel. For instance, the values $\tilde{f}$ on $\gamma_{i}$ can be defined using the partition of unity to combine the values of $f$ at the corresponding $s$-points on $\gamma^{i+1}$ and $\gamma^{i+2}$; the same argument holds for $\hat{f}$, which is constructed starting from values on $\gamma$. In practice this means that, away from the junction on $\gamma_{i}, \tilde{f}$ is defined by the value of $f_{i+1}$ or $f_{i+2}$ at the corresponding $s$-section on $\gamma^{i+1}$ or $\gamma^{i+2}$ respectively, while near the junction a smooth transition between $f_{i+1}$ and $f_{i+2}$ takes place. However, despite the particular definition of such mappings, the analysis of our model only requires that two constants $\tilde{C}, \hat{C}>0$ exist such that $\|\tilde{f}\|_{L^{2}\left(\gamma_{i}\right)} \leq \tilde{C}\|f\|_{L^{2}\left(\gamma_{i+1}\right) \times L^{2}\left(\gamma_{i+1}\right)},\|\hat{f}\|_{L^{2}\left(\gamma_{i}\right)} \leq \hat{C}\|f\|_{L^{2}(\gamma)}$. Let $\Gamma_{i, D}, \Gamma_{i, N} \subset \partial \Omega_{i}$ be respectively the Dirichlet and Neumann boundaries. Consider the following flow problem: find $\boldsymbol{p}=\left(p_{0}, p_{1}, p_{2}\right) \in H^{1}\left(\Omega_{0}\right) \times H^{1}\left(\Omega_{1}\right) \times H^{1}\left(\Omega_{2}\right)$ such that

$$
\begin{aligned}
-\nabla \cdot\left(\mathrm{K}_{i} \nabla p_{i}\right) & =q_{i} & & \text { in } \Omega_{i}, \\
p_{i} & =\overline{p_{i}} & & \text { on } \Gamma_{i, D}, \\
\mathrm{~K}_{i} \nabla p_{i} \cdot \mathbf{n}_{i} & =h_{i} & & \text { on } \Gamma_{i, N}, \\
\theta p_{i}-\alpha \mathbf{u}_{i} \cdot \mathbf{n}_{i} & =\theta \hat{P}-\alpha \hat{U}_{\mathbf{n}}+(1-\theta)(\hat{P}-\tilde{p}) & & \text { on } \gamma_{i},
\end{aligned}
$$

with $\mathbf{u}_{i}=\mathrm{K}_{i} \nabla p_{i}$. As in Sect. 3, the coercivity of the corresponding bilinear form $a$ is easily obtained for $\theta \in[0,1]$ big enough (depending on the value of $\tilde{C}, \hat{C}$ ).

The critical issue is the reduced model for the network of fractures, whose unknowns are $\mathbf{U}=\left(\mathbf{U}_{0}, \mathbf{U}_{1}, \mathbf{U}_{2}\right) \in H^{1}\left(\gamma^{1}\right) \times H^{1}\left(\gamma^{2}\right) \times H^{1}\left(\gamma^{2}\right)$ and $P=\left(P_{0}, P_{1}, P_{2}\right) \in$ $L^{2}(\gamma)=L^{2}\left(\gamma^{1}\right) \times L^{2}\left(\gamma^{2}\right) \times L^{2}\left(\gamma^{2}\right)$, and in particular the coupling conditions at the junction.

In the sequel, we will denote by a star* any quantity evaluated at the junction. We propose to treat the junction as a lumped 0D element, featuring a hydraulic pressure $P^{*}$. This pressure is actually the Lagrange multiplier associated to the mass 
balance equation at the junction. Assuming that the tangent unit vectors $\boldsymbol{\tau}_{i}^{*}$ at the endpoints of each branch $\gamma^{i}$ corresponding to the junctions are directed outwards, the mass balance reads

$$
\sum_{i \in I} \delta_{i} U_{i, \tau}^{*}=0
$$

i.e. the net flow rate entering the $0 \mathrm{D}$ element is zero. Moreover, the balance of the momentum requires that the stress at the junction equals $P^{*}$, that is

$$
-\epsilon_{i} \frac{\partial \mathbf{U}_{i}}{\partial s}+P_{i}=P^{*}, \quad \forall i \in I .
$$

In this way, the junction boundary of each branch is treated as a Neumann boundary, the prescribed stress being defined by the incompressibility condition at the junction. Accordingly, the vector $\mathbf{H}$ containing the prescribed stresses at the junction boundaries in the weak formulation of the reduced model (3.19), is given by $\mathbf{H}=\mathbf{H}^{*}=\left[P^{*}, 0\right]^{T}$. In a compact form, the weak formulation of the reduced model (3.19) for a single fracture is modified straightforwardly as follows: find $\mathbf{U}=\left(\mathbf{U}_{0}, \mathbf{U}_{1}, \mathbf{U}_{2}\right) \in H^{1}\left(\gamma^{0}\right) \times$ $H^{1}\left(\gamma^{1}\right) \times H^{1}\left(\gamma^{2}\right), P=\left(P_{0}, P_{1}, P_{2}\right) \in L^{2}(\gamma) \equiv L^{2}\left(\gamma^{0}\right) \times L^{2}\left(\gamma^{1}\right) \times L^{2}\left(\gamma^{2}\right)$ and $P^{*} \in \mathbb{R}$ such that

$$
\begin{aligned}
\int_{\gamma} \delta & \frac{\partial}{\partial s} U_{\boldsymbol{\tau}} R d s+\int_{\gamma} \delta \mathbf{M U} \cdot \mathbf{V} d s+\int_{\gamma} \delta \varepsilon \frac{\partial \mathbf{U}}{\partial s} \cdot \frac{\partial \mathbf{V}}{\partial s} d s \\
& -\int_{\gamma} \delta \frac{\partial}{\partial s} V_{\boldsymbol{\tau}} P d s+\int_{\gamma} \frac{2}{\alpha} P R d s \\
& -P^{*}\left(\sum_{i \in I} \delta_{i} V_{i, \boldsymbol{\tau}}^{*}\right)+\left(\sum_{i \in I} \delta_{i} U_{i, \boldsymbol{\tau}}^{*}\right) R^{*}=L(\mathbf{V}, R, \boldsymbol{p} ; \delta, \alpha, Q, \mathbf{G}, \mathbf{H}),
\end{aligned}
$$

for each $\mathbf{V}=\left(\mathbf{V}_{0}, \mathbf{V}_{1}, \mathbf{V}_{2}\right) \in H^{1}\left(\gamma^{0}\right) \times H^{1}\left(\gamma^{1}\right) \times H^{1}\left(\gamma^{2}\right), R=\left(R_{0}, R_{1}, R_{2}\right) \in$ $L^{2}(\gamma) \equiv L^{2}\left(\gamma^{0}\right) \times L^{2}\left(\gamma^{1}\right) \times L^{2}\left(\gamma^{2}\right)$ and $R^{*} \in \mathbb{R}$, where $L$ is a linear functional w.r.t. $\mathbf{V}, R$ and $\boldsymbol{p}$, depending on the parameters $\delta, \alpha, Q, \mathbf{G}$ and $\mathbf{H}$. Notice that the integrals on $\gamma$ are meant to be the sum of integrals on each branch $\gamma^{i}$. It is easy to see that, for small $\delta$, Theorem 1 can still be invoked to obtain the well-posedness of the coupled problem.

\section{Numerical simulations}

In this section we present the results of numerical simulations on a test problem that refers to the simple geometry made of two unitary squares separated by a fracture of width $\delta$ represented in Fig. 4. Consider the reduced model for a fracture in a bidimensional porous media introduced in Sect. 3. We suppose that $\Omega_{1}$ and $\Omega_{2}$ contain 
Fig. 4 Reference domain for the test problem

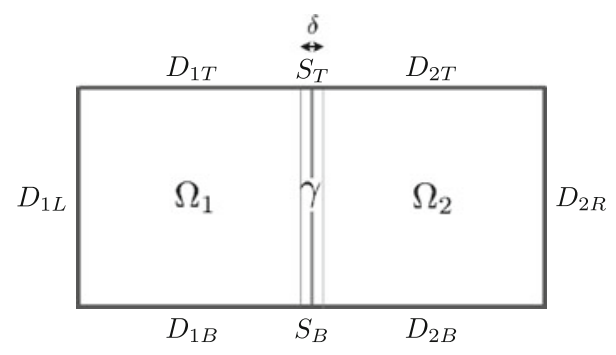

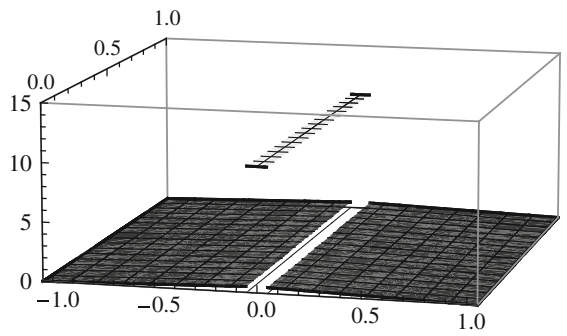

(a)

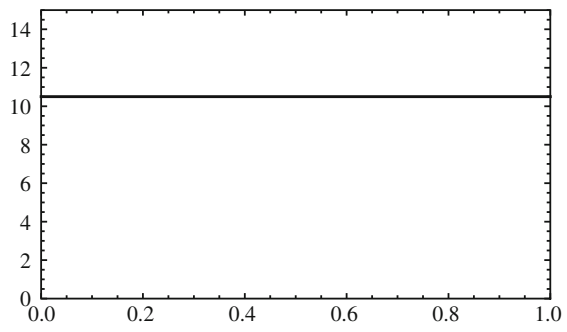

(c)

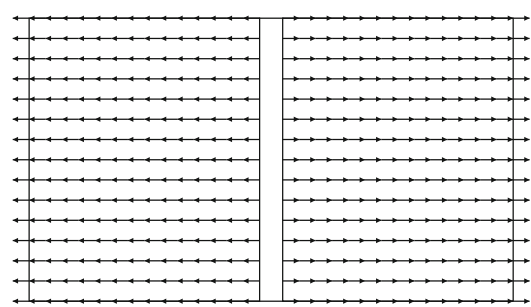

(b)

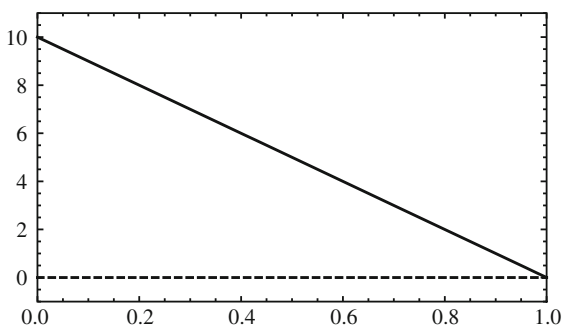

(d)

Fig. 5 a Pressure fields in $\Omega_{1}, \Omega_{2}$ and $\Omega_{f}$ for the "Symmetric Irrigation" test problem. Pressure in $\Omega_{f}$ is plotted under the assumption that it is a constant function over any given transversal section. b Velocity fields in $\Omega_{1}$ and $\Omega_{2}$. c Average pressure along $\gamma$. d Average tangential velocity (solid line) and the average normal velocity (dashed line) along $\gamma$. Parameters for the simulation are: $\delta=0.1, \varepsilon=1, \theta=1, k_{1}=1$, $k_{2}=1, p_{D_{1 L}}=0, p_{D_{2 R}}=0, U_{\tau_{S_{B}}}=10, U_{\tau_{S_{T}}}=0$ and $\mathrm{M}=0$

an isotropic porous medium with permeability tensors $k_{1} I$ and $k_{2} I$, respectively. In the following test cases, if not otherwise specified, we adopt the simpler Stokes model for describing fluid flow in the fracture (referring to Sect. 3, this implies $M=0$ ) and we suppose there are no external forces or mass sources. Dirichlet boundary conditions for pressure are imposed on segments $D_{1 L}$ and $D_{2 R}$, whereas Dirichlet boundary condition for mean tangential velocity and homogeneous Neumann conditions for mean normal strain are enforced on $S_{T}$ and $S_{B}$. On the remaining part of the boundary, either Dirichlet boundary conditions or homogeneous Neumann boundary conditions are imposed, depending on the test case considered.

A monolithic direct solver (UMFPACK) was used to solve the algebraic system (5.1). All the simulations have been performed using the free software FreeFem++ 


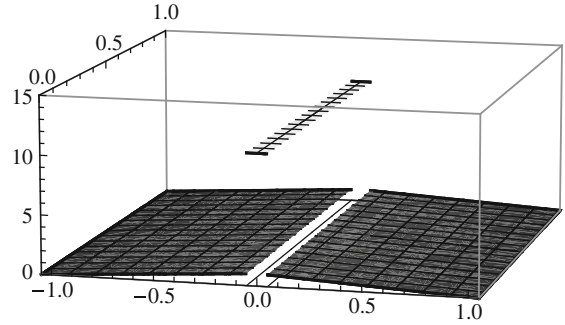

(a)

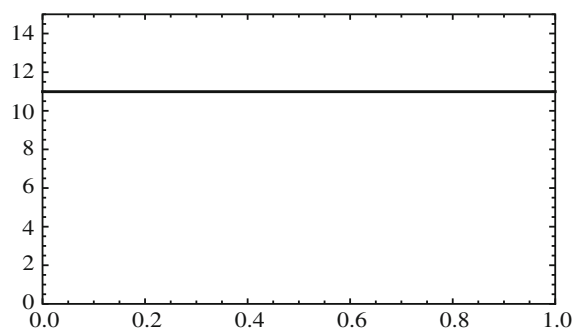

(c)

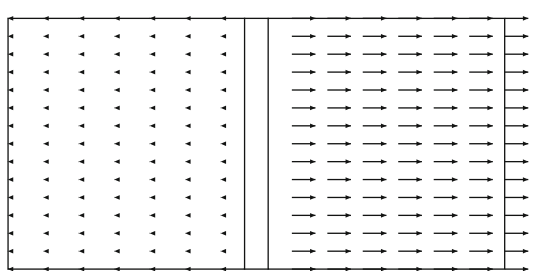

(b)

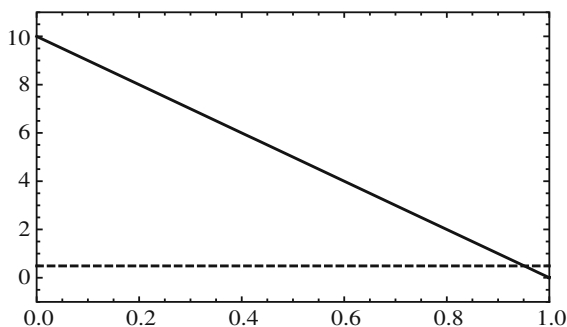

(d)

Fig. 6 a Pressure fields in $\Omega_{1}, \Omega_{2}$ and $\Omega_{f}$ for the "Symmetric Irrigation" test problem. Pressure in $\Omega_{f}$ is plotted under the assumption that it is a constant function over any given transversal section. b Velocity fields in $\Omega_{1}$ and $\Omega_{2}$. c Average pressure along $\gamma$. d Average tangential velocity (solid line) and the average normal velocity (dashed line) along $\gamma$. Parameters for the simulation are: $\delta=0.1, \varepsilon=1, \theta=1, k_{1}=0.01$, $k_{2}=1, p_{D_{1 L}}=0, p_{D_{2 R}}=0, U_{\tau_{S_{B}}}=10, U_{\tau_{S_{T}}}=0$ and $\mathrm{M}=0$

version 3.9. ${ }^{3}$ Classical unstructured FEM meshes for the solution of the problem are automatically built by the software using Delaunay triangulation and 50 nodes for each of the square edges for a total of 6789 degrees of freedom. In case of comparison with the unreduced model the exact solution is obtained through FEM on a very fine grid (200 nodes for each of the square edges and 50 nodes for fracture shorter edges for a total of 140528 degrees of freedom). To assemble the FE matrices for the reduced model in the fracture we used the special emptymesh command of the software.

Remark 9 In case of a linear fracture in a bi-dimensional domain, our analysis still holds when $\gamma_{1}$ and $\gamma_{2}$ coincide. Notice that this simplification cannot be applied neither in the case of a curved fracture nor in that of a vessel in a three dimensional domain.

\subsection{Test case 1: irrigation}

In this test case we impose a flux entering in the fracture through $S_{B}$ equal to 10 , a homogeneous Dirichlet condition for the pressure on $D_{1 L}$ and $D_{2 R}$ and for the tangential velocity on $S_{T}$, and homogeneous Neumann boundary conditions on the pressure on the remaining part of the boundary. If the pressures imposed on $D_{1 L}$ and $D_{2 R}$

\footnotetext{
3 For more details see http://www.freefem.org/ff++/.
} 


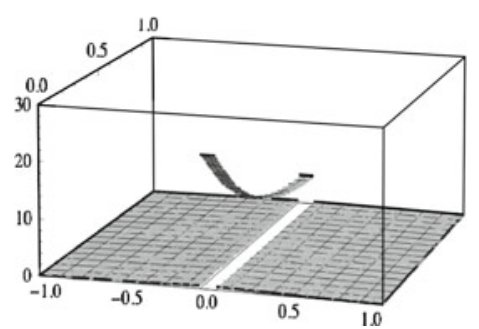

(a)

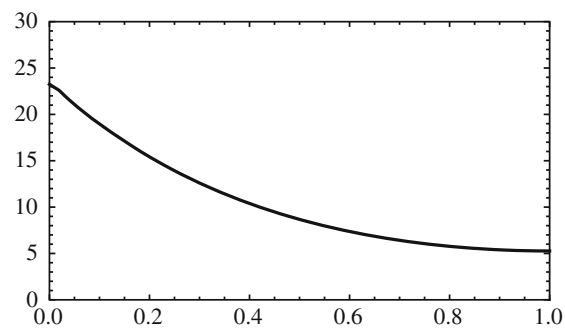

(c)

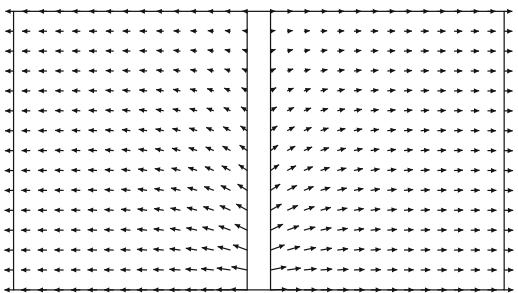

(b)

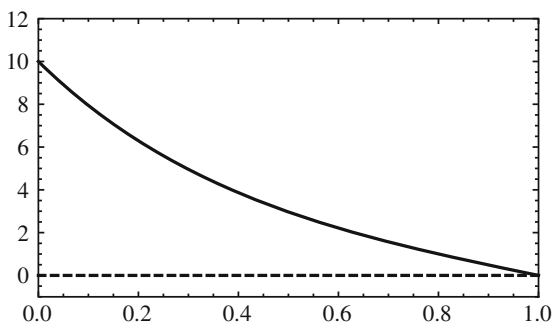

(d)

Fig. 7 a Pressure fields in $\Omega_{1}, \Omega_{2}$ and $\Omega_{f}$ for the "Brinkman" test problem. Pressure in $\Omega_{f}$ is plotted under the assumption that it is a constant function over any given transversal section. b Velocity fields in $\Omega_{1}$ and $\Omega_{2}$. c Average pressure along $\gamma$.d Average tangential velocity (solid line) and the average normal velocity (dashed line) along $\gamma$. Parameters for the simulation are: $\delta=0.1, \varepsilon=1, \theta=1, k_{1}=1, k_{2}=1, p_{D_{1 L}}=0$, $p_{D_{2 R}}=0, U_{\tau_{S_{B}}}=10, U_{\tau_{S_{T}}}=0$ and $\mathrm{M}=10 \mathrm{I}$

are equal, and so are the values $k_{1}$ and $k_{2}$, the solution is expected to be symmetric. Besides, as we supposed there is a flow entering through the bottom side of the fracture but not exiting from the top side of the fracture, it is reasonably guessed that the fluid exits the lateral walls of the fracture and propagates into the porous medium. This behaviour is correctly reproduced by our reduced model as shown in Fig. 5. Moreover we would expect that, if the the permeability in one of the two subdomains containing the porous medium is much higher than in the other one, the fluid will flow at a higher rate in the domain with higher permeability. Even this situation is correctly reproduced by the reduced model, as shown in Fig. 6 .

\subsection{Test case 2: Brinkman}

In this test case we consider the same set of boundary conditions used in the previous test case but we substitute the reduced Stokes model in the fracture with the Brinkman one. Particularly we adopted $\mathrm{M}=10 \mathrm{I}$ as the mass matrix of the Brinkman model. As in the previous case the solution is expected to be symmetric but nonlinear pressure and tangential velocity are expected in the fracture due to the presence of the dissipative term. This behaviour is correctly reproduced by our reduced model as shown in Fig. 7. 


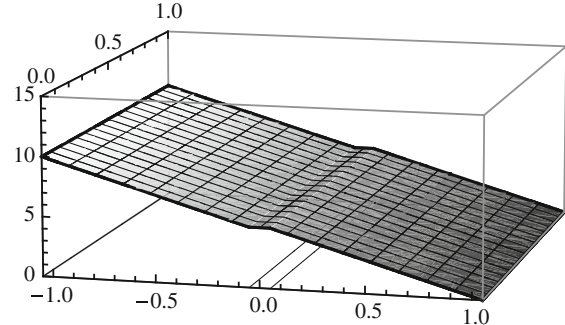

(a)

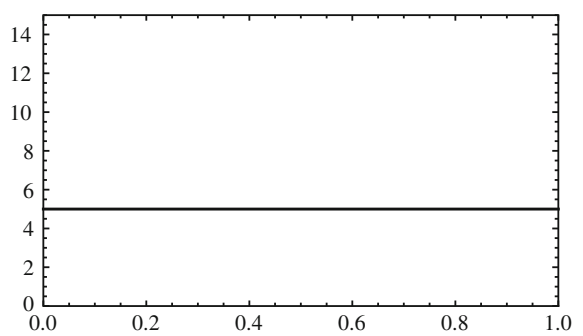

(c)

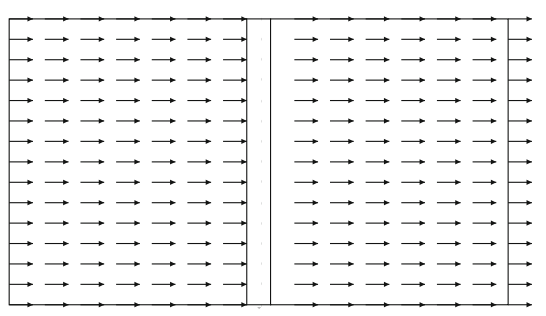

(b)

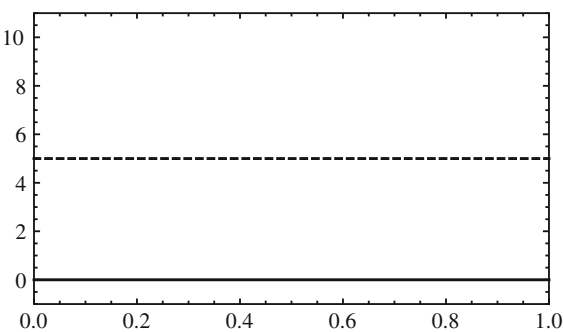

(d)

Fig. 8 a Pressure fields in $\Omega_{1}, \Omega_{2}$ and $\Omega_{f}$ for the "Symmetric Irrigation" test problem. Pressure in $\Omega_{f}$ is plotted under the assumption that it is a constant function over any given transversal section. b Velocity fields in $\Omega_{1}$ and $\Omega_{2}$. c Average pressure along $\gamma$. d Average tangential velocity (solid line) and the average normal velocity (dashed line) along $\gamma$. Parameters for the simulation are: $\delta=0.1, \varepsilon=1, \theta=1, k_{1}=1$, $k_{2}=1, p_{D_{1 L}}=10, p_{D_{2 R}}=0, U_{\tau_{S_{B}}}=0, U_{\tau_{S_{T}}}=0$ and $\mathrm{M}=0$

\subsection{Test case 3: filtration in a porous medium in the presence of a fracture}

In this test case we impose homogeneous Dirichlet condition for mean tangential velocity on $S_{B}$ and $S_{T}$ and for pressure on $D_{2 R}$, whereas we impose a positive constant pressure equal to 10 on $D_{1 L}$. We expect a continuous flux between $D_{1 L}$ and $D_{2 R}$ due to the difference of pressure at the edges. This behaviour is correctly reproduced by the reduced model as shown in Fig. 8.

\subsection{Test case 4: comparison with the results obtained from the unreduced model}

We now compare the behaviour of the reduced model and that of the unreduced one for different values of the width of the fracture $\delta(0.2,0.1,0.05,0.025$ e 0.0125$)$ and the grid size $h(0.2,0.1,0.05,0.025,0.0125)$. Consider again the geometry reported in Fig. 4 with the following parameters: $\varepsilon=1, \theta=1, k_{1}=0.01, k_{2}=1, p_{D_{1 L}}=20$, $p_{D_{2 B}}=20, p_{D_{2 T}}=-20(x+\delta / 2), p_{D_{2 R}}=10, p_{D_{2 B}}=10(x-\delta / 2), p_{D_{2 T}}=10$, $U_{\tau_{S_{B}}}=10$ and $U_{\tau_{S_{T}}}=5$. We considered the relative error for the $L^{2}$ and $H^{1}$ norms of pressure in $\Omega_{1} \cup \Omega_{2}, L^{2}$ and $H^{1}$ norms of mean tangential and normal velocity in the fracture, and the $L^{2}$ norm of pressure in the fracture. We can highlight the following behaviours: 


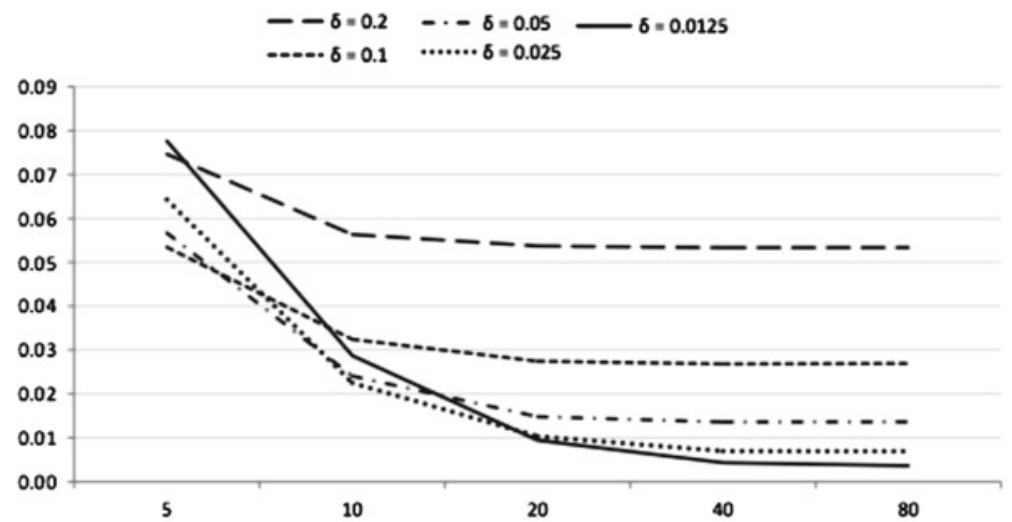

(a)

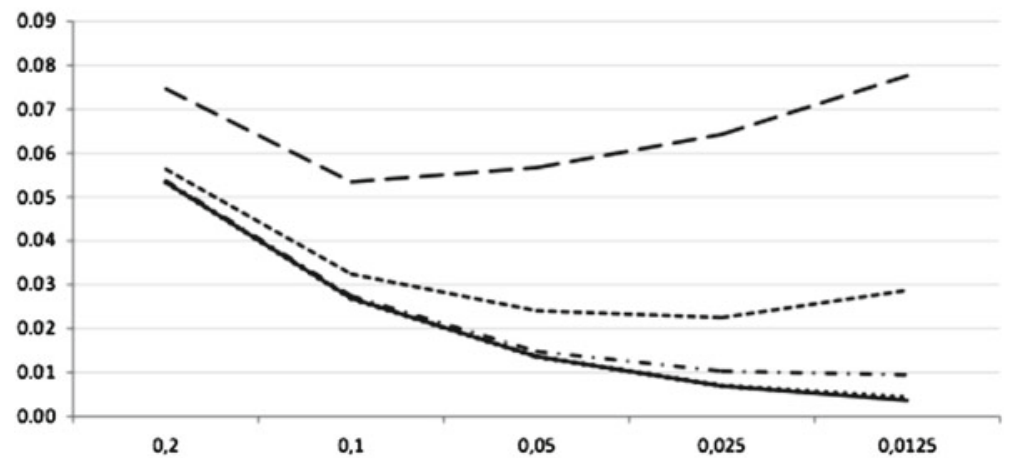

(b)

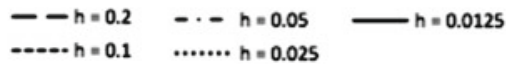

Fig. 9 a Relative error for the $L^{2}$ norm of pressure in $\Omega_{1} \cup \Omega_{2}$ for the problem presented in Sect. 7.4 depending on $\delta$ for fixed $h$. b Relative error for the $L^{2}$ norm of pressure in $\Omega_{1} \cup \Omega_{2}$ for the problem presented in Sect. 7.4 depending on $h$ for fixed $\delta$

- The relative error is decreasing when $h$ is increased but reaches a plateau (a lower threshold) due to the intrinsic error of the reduced model. The value of the plateau depends on the width $\delta$ of the fracture and decreases when $\delta$ decreases.

- For fixed $h$, the relative error decreases with $\delta$, however if $h$ is too large the error may instead increase.

The same kind of behaviour occurs for all the norms considered. In case $h$ is small enough the relative error is under $10-15 \%$ in case of large fracture width $(\delta=0.2$, i.e. one fifth of the characteristic dimension of the porous media) and large $h(0.1$ or 0.2 ). The relative error decreases significantly to $0.1-5 \%$ in case of small fracture width $(\delta=0.025$, i.e. $1 / 40$ of the characteristic dimension of the porous media) and small $h(0.0125)$. As an example in Fig. 9 we presented the typical dependance of the relative error (in this case referred to the $L^{2}$ norm of pressure in $\Omega_{1} \cup \Omega_{2}$ ) on $\delta$ and $h$. 


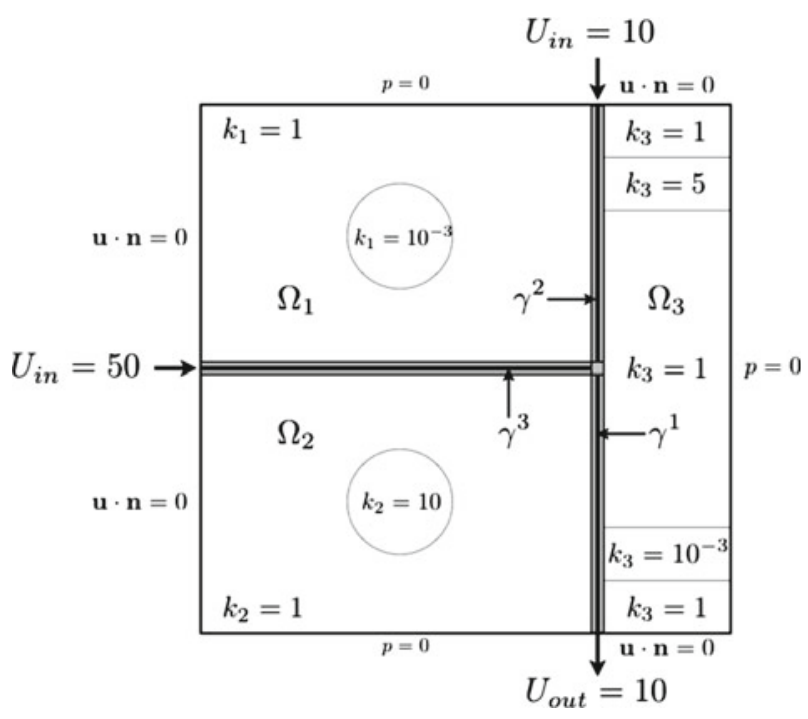

Fig. 10 Reference geometry, permeability values and boundary conditions for the T-Junction test case

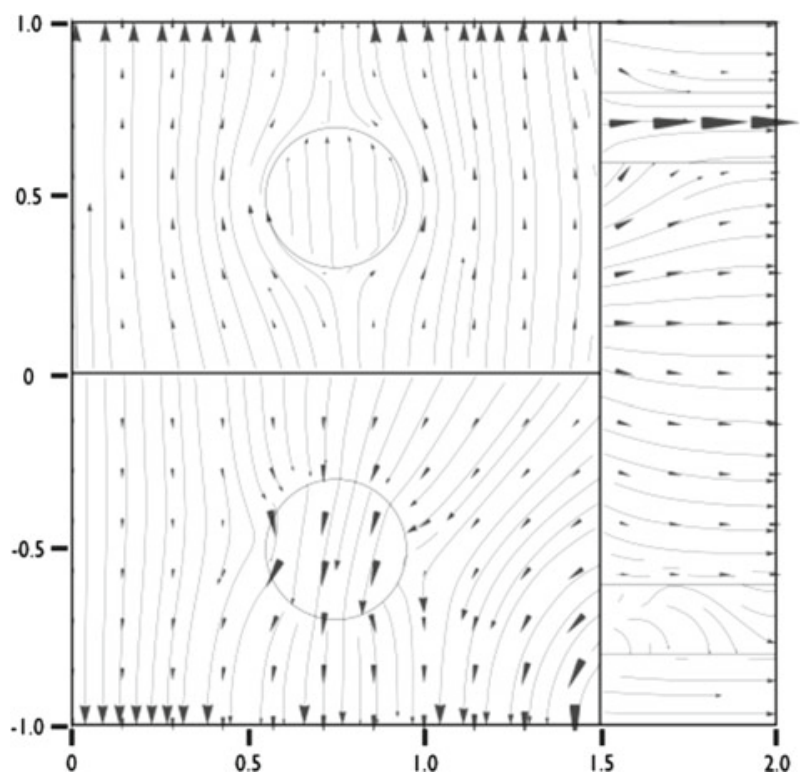

Fig. 11 Velocity fields and streamlines in the porous media for the T-Junction test case

Remark 10 For the test cases presented in Sects. 7.1, 7.2 and 7.3 the results of the reduced model are excellent. Relative errors for quantities that are non-null in the exact solution are below $0.005 \%$ even in the case of large fracture $(\delta=0.1$, i.e. $1 / 10$ of the characteristic dimension of the porous media) and large $h(0.2)$. This behaviour 


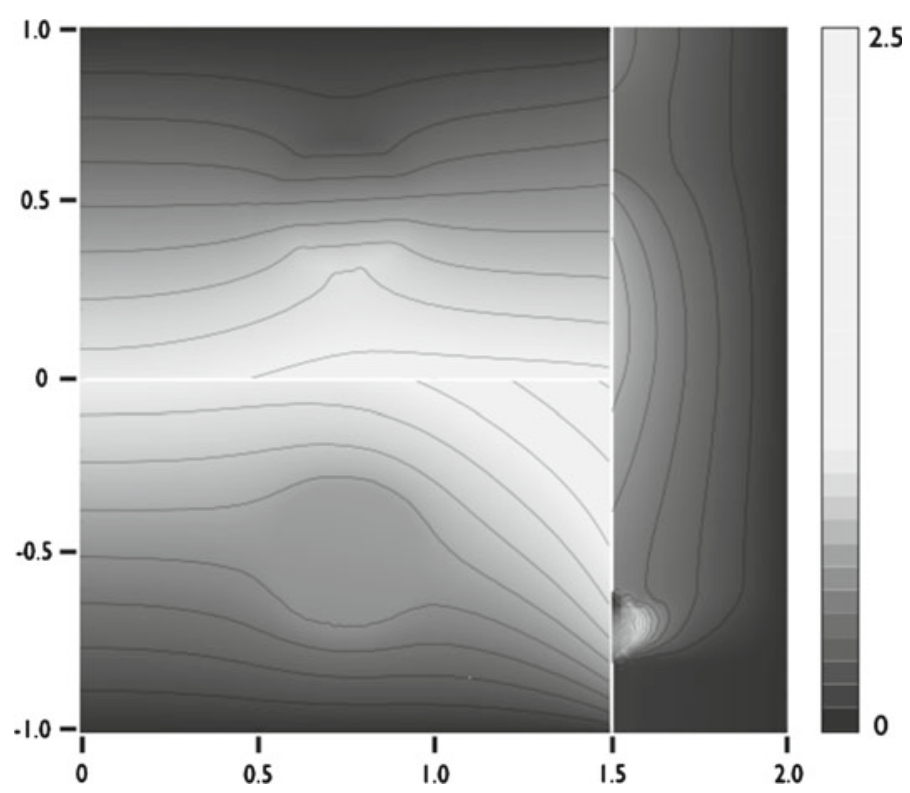

Fig. 12 Density plot and contourline of pressure in the porous media for the T-Junction test case

is due to the fact that the solution (pressure in $\Omega_{1}$ and $\Omega_{2}$ and mean pressure, mean normal velocity and mean tangential velocity in the fracture) of the exact (unreduced) problem are linear functions.

\subsection{Test case 5: T-junction}

We now present the results obtained for the problem illustrated in Fig. 10.

Areas featuring different permeability values are considered inside the porous media; besides, the presence of a T-Junction fractures network allows us to assess the performance of the extension of our model to the case of a network that has been introduced in Sect. 6. The parameters for this simulation are $\delta_{i}=0.05, \varepsilon=1$ and $\theta=1$. As shown in Fig. 11, the fluid exits the fractures and propagates into the porous media. This is due to the fact that the Dirichlet boundary conditions for $U_{\tau}$ are not balanced $(50+10$ entering and 10 exiting the fracture). In the porous media the fluid flows following different paths depending on the permeability distribution: high permeability zones tend to attract fluid from the neighbour regions, whereas low permeability or impermeable regions tend to deviate the fluid apart from themselves. A density plot for the pressure in the porous media is shown in Fig. 12. Figure 13a-c shows the mean tangential velocity inside the three fractures. Note that in Fig. 13a-c a positive value of the mean tangential velocity means that the flow is directed towards the junction whereas a negative value indicates that the flow is directed towards the external boundaries. Figure $14 \mathrm{a}-\mathrm{c}$ shows instead the mean pressure inside the three fractures that is almost constant apart from a small area in proximity of the low permeability channel present in domain $\Omega_{3}$. With further computations on the numerical solution it is also possible to verify 


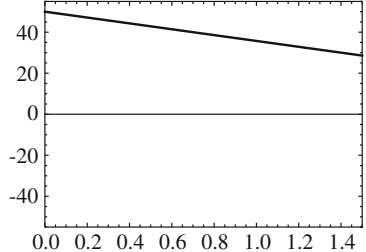

(a)

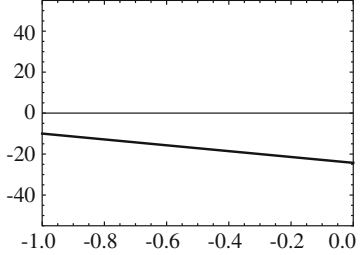

(b)

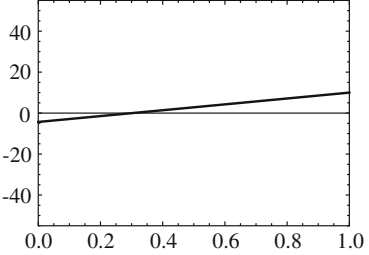

(c)

Fig. 13 Mean tangential velocities in the fractures $\gamma^{1}(\mathbf{a}), \gamma^{2}(\mathbf{c})$ and $\gamma^{3}(\mathbf{b})$. Note that $\gamma^{1}$ separates the domain $\Omega_{2}$ from the domain $\Omega_{3}, \gamma^{2}$ the domain $\Omega_{1}$ from $\Omega_{3}$, and $\gamma^{3}$ the domain $\Omega_{1}$ from $\Omega_{2}$. Positive values indicate that the mean velocity is directed towards the T-Junction

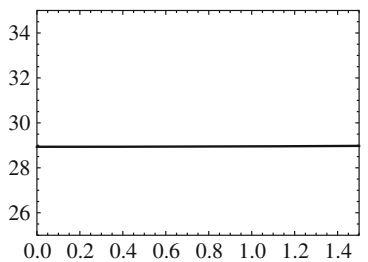

(a)

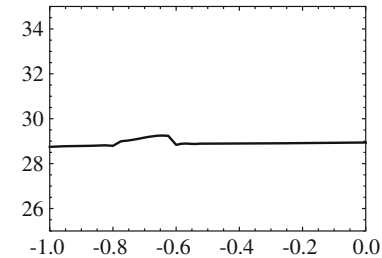

(b)

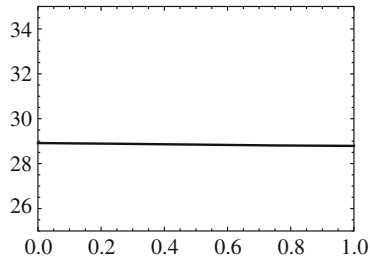

(c)

Fig. 14 Mean pressure values in the fractures $\gamma^{1}(\mathbf{a}), \gamma^{2}(\mathbf{c})$ and $\gamma^{3}(\mathbf{b})$

that the mass balance Eq. (6.1) and the stress balance Eq. (6.2) at the T-Junction are satisfied.

\section{Conclusions}

In this work we proposed a multiscale model for incompressible fluid flow in porous media with fractures, based on the coupling of Darcy and Brinkman equations. This model significantly differs from previously proposed models, since (i) it allows the treatment of viscous flow inside fractures, and (ii) the interfaces between the fracture and the porous domain are kept separated, which makes simpler the analysis and the determination of the coupling conditions in several cases. The well-posedness of the problem is asserted under the assumption that the fracture or the vessels are small enough. Suitable numerical approaches to effectively solve the problem have been provided. Moreover, the simulations highlight the potential of this model to reproduce the relevant aspects related to the presence of fractures.

Acknowledgments This work has been partially supported by the ERC Advanced Grant N.227058 Mathematical Modelling and Simulation of the Cardiovascular System (MATHCARD). The authors thank the anonymous referee for many remarks and criticisms that helped us to improve our paper.

\section{References}

1. Ainsworth, M., Sherwin, S.: Domain decomposition preconditioners for $p$ and $h p$ finite element approximation of Stokes equations. Comput. Methods Appl. Mech. Eng. 175(3-4), 243-266 (1999) 
2. Alboin, C., Jaffré, J., Roberts, J.E., Serres, C.: Modeling fractures as interfaces for flow and transport in porous media. In: Fluid Flow and Transport in Porous Media: Mathematical and Numerical Treatment (South Hadley, MA, 2001). Contemp. Math., vol. 295, pp. 13-24. Amer. Math. Soc., Providence (2002)

3. Angot, P., Boyer, F., Hubert, F.: Asymptotic and numerical modelling of flows in fractured porous media. Math. Model. Numer. Anal. 43(2), 239-275 (2009)

4. Averbuch, A., Israeli, M., Vozovoi, L.: A fast Poisson solver of arbitrary order accuracy in rectangular regions. SIAM J. Sci. Comput. 19(3), 933-952 (1998)

5. Beavers, G., Joseph, D.: Boundary conditions at a naturally permeable wall. J. Fluid Mech. 30, 197-207 (1967)

6. Bernhardt, P.A., Brackbill, J.U.: Solution of elliptic equations using fast Poisson solvers. J. Comput. Phys. 53(3), 382-394 (1984)

7. Bogdanov, I.I., Mourzenko, V.V., Thovert, J.F., Adler, P.M.: Two-phase flow through fractured porous media. Phys. Rev. E 68(2), 026,703 (2003). doi:10.1103/PhysRevE.68.026703

8. Brenner, S.C.: A two-level additive Schwarz preconditioner for the stationary Stokes equations. Adv. Comput. Math. 4(1-2), 111-126 (1995)

9. Cao, Z.H.: Constraint Schur complement preconditioners for nonsymmetric saddle point problems. Appl. Numer. Math. 59(1), 151-169 (2009)

10. Carvalho, L.M., Giraud, L., Le Tallec, P.: Algebraic two-level preconditioners for the Schur complement method. SIAM J. Sci. Comput. 22(6), 1987-2005 (2000)

11. Casarin, M.A.: Schwarz preconditioners for the spectral element discretization of the steady Stokes and Navier-Stokes equations. Numer. Math. 89(2), 307-339 (2001)

12. Chang, S.C.: Solution of elliptic PDEs by fast Poisson solvers using a local relaxation factor. J. Comput. Phys. 67(1), 91-123 (1986)

13. D'Angelo, C., Quarteroni, A.: On the coupling of 1d and 3D diffusion-reaction equations. Application to tissue perfusion problems. Math. Models Methods Appl. Sci. 18(8), 1481-1504 (2007)

14. Darcy, H.: Les Fontaines Publiques de la Ville de Dijon. Dalmont (1856)

15. Deuflhard, P., Hochmuth, R.: Multiscale analysis of thermoregulation in the human microvascular system. Math. Methods Appl. Sci. 27(8), 971-989 (2004)

16. Discacciati, M., Quarteroni, A.: Navier-Stokes/Darcy coupling: modeling, analysis, and numerical approximation. Rev. Mater. Comput. 22(2), 315-426 (2009)

17. Elman, H.C.: Approximate Schur complement preconditioners on serial and parallel computers. SIAM J. Sci. Stat. Comput. 10(3), 581-605 (1989)

18. Ern, A., Guermond, J.L.: Theory and Practice of Finite Elements. Springer, Berlin (2004)

19. Formaggia, L., Gerbeau, J.F., Nobile, F., Quarteroni, A.: On the coupling of 3D and 1D Navier-Stokes equations for flow problems in compliant vessels. Comput. Methods Appl. Mech. Eng. 191(6-7), 561-582 (2001)

20. Formaggia, L., Quarteroni, A., Veneziani, A.: Cardiovascular Mathematics. Springer, Milan (2009)

21. Frih, N., Roberts, J.E., Saada, A.: Modeling fractures as interfaces: a model for Forchheimer fractures. Comput. Geosci. 12(1), 91-104 (2008)

22. Giraud, L., Tuminaro, R.S.: Schur complement preconditioners for anisotropic problems. IMA J. Numer. Anal. 19(1), 1-18 (1999)

23. Glowinski, R.: On a new preconditioner for the Stokes problem. Mat. Apl. Comput. 6(2), 123-140 (1987)

24. Jaffré, J.: Numerical calculation of the flux across an interface between two rock types of a porous medium for a two-phase flow. In: Hyperbolic Problems: Theory, Numerics, Applications (Stony Brook, NY, 1994), pp. 165-177. World Scientific, River Edge (1996)

25. Jaffré, J., Martin, V., Roberts, J.E.: Modeling fractures and barriers as interfaces for flow in porous media. SIAM J. Sci. Comput. 26, 1667-1691 (2005)

26. Jager, W., Mikelic, A.: On the interface boundary condition of Beavers, Joseph and Saffman. SIAM J. Appl. Math. 60, 1111-1127 (2000)

27. Lee, D.: Boundary collocation fast Poisson solver on irregular domains. Korean J. Comput. Appl. Math. 8(1), 27-44 (2001)

28. Lesinigo, M.: Analisi di modelli multiscala per fluidi in mezzi porosi con fratture. Master thesis, Politecnico di Milano (2007/2008)

29. Liniger, W.: A quasi-direct fast Poisson solver for general regions. In: Proceedings of the 3rd International Congress on Computational and Applied Mathematics (Leuven, 1988), vol. 28, pp. 25-47 (1989) 
30. Martin, V., Jaffré, J., Roberts, J.E.: Modeling fractures and barriers as interfaces for flow in porous media. SIAM J. Sci. Comput. 26(5), 1667-1691 (2005)

31. McKenney, A., Greengard, L., Mayo, A.: A fast Poisson solver for complex geometries. J. Comput. Phys. 118(2), 348-355 (1995)

32. Moriya, K., Nodera, T.: Computing the preconditioner for the Schur complement. ANZIAM J. 46(C), C394-C408 (2004/05)

33. Onana, A., Kwankam, S.Y., Zoue, E.: A fast Poisson solver. Period. Math. Hungar. 28(2), 89-101 (1994)

34. Oswald, P.: An optimal multilevel preconditioner for solenoidal approximations of the two-dimensional Stokes problem. IMA J. Numer. Anal. 18(2), 207-228 (1998)

35. Quarteroni, A.: Numerical Models for Differential Problems. Springer, Milan (2009)

36. Quarteroni, A., Veneziani, A.: Analysis of a geometrical multiscale model based on the coupling of ODEs and PDEs for blood flow simulations. Multiscale Model. Simul. 1(2), 173-195 (2003)

37. Roberts, J., Thomas, J.: Handbook of Numerical Analysis, vol. 2. Elsevier, Amsterdam (1990)

38. Saad, Y.: Schur complement preconditioners for distributed general sparse linear systems. In: Domain Decomposition Methods in Science and Engineering XVI. Lect. Notes Comput. Sci. Eng., vol. 55, pp. 127-138. Springer, Berlin (2007)

39. Saffman, P.: On the boundary condition at the interface of a porous medium. Stud. Appl. Math. 1, 93-101 (1971)

40. Schröder, J., Trottenberg, U., Witsch, K.: On fast Poisson solvers and applications. In: Numerical Treatment of Differential Equations (Proc. Conf., Math. Forschungsinst., Oberwolfach, 1976), pp. 153-187. Lecture Notes in Math., vol. 631. Springer, Berlin (1978)

41. Sharipov, R.A.: Course of Differential Geometry. Bashkir State University (1996)

42. Silvester, D., Wathen, A.: Fast iterative solution of stabilised Stokes systems. II. Using general block preconditioners. SIAM J. Numer. Anal. 31(5), 1352-1367 (1994)

43. Swarztrauber, P.N.: Fast Poisson solvers. In: Studies in Numerical Analysis, MAA Stud. Math., vol. 24, pp. 319-370. Math. Assoc. America, Washington (1984)

44. Wathen, A., Silvester, D.: Fast iterative solution of stabilised Stokes systems. I. Using simple diagonal preconditioners. SIAM J. Numer. Anal. 30(3), 630-649 (1993) 